\title{
EEG Biofeedback as a Treatment for Substance Use Disorders: Review, Rating of Efficacy, and Recommendations for Further Research
}

\author{
Tato M. Sokhadze · Rex L. Cannon • \\ David L. Trudeau
}

Published online: 24 January 2008

(C) Springer Science+Business Media, LLC 2008

\begin{abstract}
Electroencephalographic (EEG) biofeedback has been employed in substance use disorder (SUD) over the last three decades. The SUD is a complex series of disorders with frequent comorbidities and EEG abnormalities of several types. EEG biofeedback has been employed in conjunction with other therapies and may be useful in enhancing certain outcomes of therapy. Based on published clinical studies and employing efficacy criteria adapted by the Association for Applied Psychophysiology and Biofeedback and the International Society for Neurofeedback and Research, alpha theta training-either alone for alcoholism or in combination with beta training for stimulant and mixed substance abuse and combined with residential treatment programs, is probably efficacious. Considerations of further research design taking these factors into account are discussed and descriptions of contemporary research are given.
\end{abstract}

Keywords Neurofeedback · Neurotherapy · EEG biofeedback - Quantitative EEG - Substance use disorder . Alcoholism · ERP · Cognitive-behavioral treatment

\footnotetext{
T. M. Sokhadze $(\square)$

Department of Psychiatry and Behavioral Sciences, University

of Louisville School of Medicine, Louisville, KY, USA

e-mail: tato.sokhadze@louisville.edu

R. L. Cannon

Department of Psychology, The University of Tennessee, Knoxville, TN 37996, USA

e-mail: rcannon2@utk.edu

D. L. Trudeau

Department of Family and Community Health, School of Health

Sciences, University of Minnesota, Minneapolis, MN, USA

e-mail: trude003@maroon.tc.umn.edu
}

\section{Introduction}

Substance use disorders (SUD) include disorders related to the taking of a drug of abuse (including alcohol), and represent the most common psychiatric conditions (APA 2000) resulting in serious impairments in cognition and behavior. Acute and chronic drug abuse results in significant alteration of the brain activity detectable with quantitative electroencephalography (qEEG) methods. The treatment of addictive disorders by electroencephalographic (EEG) biofeedback (or neurofeedback, as it is often called) was first popularized by the work of Eugene Peniston (Peniston and Kulkosky 1989, 1990, 1991) and became popularly known as the Peniston Protocol. This approach employed independent auditory feedback of two slow brain wave frequencies, alpha $(8-13 \mathrm{~Hz})$ and theta (4-8 Hz) in an eyes closed condition to produce a hypnagogic state. The patient was taught prior to neurofeedback to use what amounts to success imagery (beingsober, refusing offers of alcohol, living confidently, and happy) as they drifted down into an alpha-theta state. Repeated sessions reportedly resulted in long-term abstinence and changes in personality testing. Because the method seemed to work well for alcoholics, it has been tried in subjects with cannabis dependence and stimulant dependence- - but with limited success until the work of Scott and Kaiser (Scott and Kaiser 1998; Scott et al. 2002, 2005). They described treating stimulant abusing subjects with attention-deficit type EEG biofeedback protocols, followed by the Peniston Protocol, with substantial improvement in program retention and long-term abstinence rates. This approach has become known widely as the Scott-Kaiser modification (of the Peniston Protocol).

This "white paper" on EEG biofeedback for SUD will offer an assessment of efficacy according to the 
guidelines jointly established by the Association for Applied Psychophysiology and Biofeedback (AAPB) and the International Society for Neurofeedback and Research (ISNR). Assessing the efficacy of neurofeedback for SUD involves several considerations. The first of these involves difficulties assessing the efficacy of any treatment method for SUD. Outcome benchmarks (i.e., total abstinence, improved function and quality of life) and time points of outcome (i.e., one year, two years post treatment) are not clearly established.

Outcome assessment for treatment of SUD in itself is a complex topic well beyond the scope of this article. Because different drugs of abuse are associated with different patterns of EEG abnormality, as will be discussed in detail in this article, it is difficult to assign broad-brush EEG biofeedback solutions to SUD as a whole. Any statements of efficacy will need to describe specific EEG biofeedback protocols for specific substances of abuse. Furthermore substance abuse is often mixed substance type and comorbid conditions are common and vary from subject to subject, as will also be borne out in this article. As of yet there are no gold standard medication or other treatments for the various types of SUD and efficacy of any SUD treatment method likely falls into the "possibly effective" to "probably effective" range according to the efficacy guidelines jointly established by the AAPB and ISNR. Finally, all of the studies of EEG biofeedback in SUD to date employ EEG biofeedback as an add on to cognitive behavioral or twelve step treatment regimes, so any statements of efficacy would have to acknowledge that EEG biofeedback is not a stand alone treatment for SUD.

This article is divided into several sections. In the first section after "Introduction," we review SUD prevalence and describe qEEG changes typical for the most widespread drugs of abuse (alcohol, marijuana, heroin, cocaine, and methamphetamine). The second section describes treatment studies employing EEG biofeedback in SUD. Studies that have used the Peniston Protocol are described first, along with critical commentaries of these studies. In the second part of this section, a description of the ScottKaiser modification is given, along with some discussion of a rationale for why this approach may be more successful with stimulant abusers. This section also describes some current research. The third section assesses efficacy of the Peniston Protocol and the Scott-Kaiser modification. The fourth section takes a look at the clinical implications of comorbidities in neurobiofeedback treatment of alcohol and drug abuse. The fifth section discusses the clinical implications of standard cognitive-behavioral therapies in SUD treatment and reviews the rationale for the application of qEEG-guided neurofeedback intervention in SUD in conjunction with these therapies. The final section summarizes findings in qEEG and neurofeedback in SUD and additionally proposes further directions for clinical research in this area.

This article represents an update of earlier reviews (Trudeau 2000, 2005a, b) of EEG biofeedback for addictive disorders extended with a review on qEEG in SUD. This review is presented as one of a series of papers in both The Journal of Neurotherapy and The Journal of Applied Psychophysiology \& Biofeedback describing and reviewing biofeedback applications for adult populations. No attempt will be made to review the fields of qEEG and neurobiofeedback generally (see current reviews by Hammond 2006; Kaiser 2006), or the field of addictive disorders generally, although some references will be made to specifics the authors feel are pertinent to a discussion of emerging concepts of qEEG as a sensitive tool for the brain function assessment in SUD, and EEG biofeedback as a treatment approach for SUD.

\section{SUD Prevalence and qEEG Changes}

Drug addiction can be described as a mental disorder with idiosyncratic behavioral, cognitive, and psychosocial features. The SUD commonly referred to as "drug addiction" is characterized by physiological dependence accompanied by the withdrawal syndrome on discontinuance of the drug use, psychological dependence with craving, the pathological motivational state that leads to the active drugseeking behavior, and tolerance, expressed in the escalation of the dose needed to achieve a desired euphoric state. Drug addiction is a chronic, relapsing mental disease that results from the prolonged effects of drugs on the brain (Dackis and O'Brain 2001; Volkow et al. 2003, 2004). Drug addiction can take control of the brain and behavior by activating and reinforcing behavioral patterns that are excessively directed to compulsive drug use (Di Chiara 1999; Gerdeman et al. 2003).

From the 11 classes of substances listed in the DSM-IV we will discuss in our review only alcohol, cannabis (marijuana), heroin, and such psychostimulants as cocaine and methamphetamine. Addiction leads to behavioral, cognitive, and social adverse outcomes that incur substantial costs to society. In 2002, it was estimated from the Substance Abuse and Mental Health Service Administration (SAMHSA 2004) that 22 million Americans have a substance abuse or dependence disorder, and 2 million of them were current cocaine users (Vocci and Ling 2005). In 2005, there were 2.4 million persons who were current cocaine users, which is more than in 2004 (SAMHSA 2006). The number of current crack users increased from 467,000 in 2004 to 682,000 in 2005. According to the 2004 revised National Survey on Drug Use and Health, nearly 12 million Americans have tried methamphetamine, and 
583,000 of them are chronic methamphetamine users (SAMHSA 2004). In 2005, an estimated 22.2 million persons aged 12 or older were classified with substance dependence or abuse in the past year $(9.1 \%$ of the population aged 12 or older). Of these, 3.3 million were classified with dependence on or abuse of both alcohol and illicit drugs, 3.6 million were dependent on or abused illicit drugs but not alcohol, and 15.4 million were dependent on or abused alcohol but not illicit drugs. There were 18.7 million persons classified with dependence on or abuse of alcohol in 2005 (7.7\%). The specific illicit drugs that had the highest levels of past year dependence or abuse in 2005 were marijuana, followed by cocaine and pain relievers. Of the 6.8 million persons aged 12 or older classified with dependence on or abuse of illicit drugs, 4.1 million were dependent on or abused marijuana in 2005 . This number represents $1.7 \%$ of the total population aged 12 or older and $59.9 \%$ of all those classified with illicit drug dependence or abuse. Marijuana was the most commonly used illicit drug (14.6 million past month users). In 2005 , it was used by $74.2 \%$ of current illicit drug users. Among current illicit drug users, $54.5 \%$ used only marijuana, $19.6 \%$ used marijuana and another illicit drug, and the remaining $25.8 \%$ used only an illicit drug other than marijuana in the past month (SAMHSA 2006).

Fatal poisoning, which include overdoses (ODs) on illicit drugs, alcohol, and medications, is the leading cause of injury death for individuals age 35-44 and the third leading cause of injury death overall, trailing motor vehicle accidents and firearm-related deaths (CDC 2004). Heroinrelated ODs have increased at an alarming rate in portions of the US and other countries (Darke and Hall 2003; Landen et al. 2003), and OD has surpassed HIV infection as the primary cause of death for heroin users. Not surprisingly, heroin is frequently associated with opioidrelated ODs, both as a single drug and in combination with other substances (CDC 2004).

Many patients seeking treatment for addiction have multiple drug dependencies and psychiatric comorbidities (Volkow and Li 2005). Information from epidemiological surveys indicates that drug addiction is a common phenomenon and is associated with significant effects on both morbidity and mortality. Large individual and societal costs of drug abuse make research and treatment of drug addiction imperative (French et al. 2000; Mark et al. 2001). Recently through intensive clinical neurophysiological research and biological psychiatric studies many specific components of cognitive, emotional, and behavioral deficits typical for SUD have been identified and investigated. However, the practical values of these cognitive neuroscience and applied psychophysiology-based treatment (e.g., neurofeedback) findings depend on a further integration of these methodological approaches.
qEEG in Substance Use Disorders

\section{EEG in Alcoholism}

EEG alterations have been described extensively in alcoholic patients (Porjesz and Begleiter 1998), but any attempt at drawing a common picture from qEEG data is difficult due to significant methodological differences, such as different definitions of frequency bands, different filtering methodology, number of channels, reference choice, etc. However, most reports of alcoholic patients agree in describing alterations mainly within the beta (Bauer 1997, 2001a; Costa and Bauer 1997; Rangaswamy et al. 2002, 2004) and/or alpha bands (Finn and Justus 1999).

The qEEG and LORETA mapping studies of detoxified alcohol-dependent patients, as compared with normal controls, showed an increase in absolute and relative beta power and a decrease in alpha and delta/theta power (Saletu et al. 2002), which is in agreement with earlier reports of low-voltage fast EEG patterns, as often encountered by visual EEG inspection (Niedermeyer and Lopes da Silva 1982). As slow activities are considered to be inhibitory, alpha activity may be viewed as an expression of normal brain functioning and fast beta activities as excitatory, the low-voltage fast desynchronized patterns may be interpreted as hyperarousal of the central nervous system (CNS) (Saletu-Zyhlarz et al. 2004). The investigations by Bauer (2001a) and Winterer et al. (1998) showed a worse prognosis for the patient group with a more pronounced frontal CNS hyperarousal. It may be hypothesized that these hyperaroused relapsing patients require more CNS sedation than abstaining ones.

The EEG maps of alcohol-dependent patients differ significantly from those of normal controls and patients suffering from other mental disorders and might be useful for diagnostic purposes (Pollock et al. 1992; Saletu et al. 2002; Saletu-Zyhlarz et al. 2004). Decreased power in slow bands in alcoholic patients may be an indicator of brain atrophy and chronic brain damage, while an increase in the beta band may be related to various factors such as medication use, family history of alcoholism, and/or hallucinations, suggesting a state of cortical hyperexcitability (Coutin-Churchman et al. 2006).

Abnormalities in resting EEG are often associated with a predisposition to development of alcoholism. Subjects with a family history of alcoholism were found to have reduced relative and absolute alpha power in occipital and frontal regions and increased relative beta in both regions compared with subjects with a negative family history of alcoholism. These results suggest that resting EEG alpha abnormalities are associated with risk for alcoholism, although their etiological significance is unclear (Finn and Justus 1999). 
Alcohol-dependent individuals have different synchronization of brain activity than light drinkers as reflected by differences in resting EEG coherence (Kaplan et al. 1985, 1988; Michael et al. 1993; Winterer et al. 2003a) and power (e.g., Bauer 2001a b; Enoch et al. 2002; Rangaswamy et al. 2002; Saletu-Zyhlarz et al. 2004). Most differences in EEG coherence and power are found in the alpha and beta bands. Non-alcoholdependent relatives of alcohol-dependent individuals also have EEG differences in alpha and beta coherence (Michael et al. 1993) and power (Bauer and Hesselbrock 2002; Finn and Justus 1999; Rangaswamy et al. 2002, 2004) as compared to subjects without alcohol-dependent relatives. This indicates that differences in functional brain activity as measured with qEEG in alcoholdependent patients not only relate to the impact of longterm alcohol intake, but possibly also to genetic factors related to alcohol dependence.

Both alcohol dependence (Schuckit and Smith 1996) and EEG patterns (Van Beijsterveldt and Van Baal 2002) are highly heritable. In addition, some genes coding for GABA receptors in the brain, which mediate the effects of alcohol, are related to certain EEG patterns (Porjesz et al. 2005; Winterer et al. 2003b). Moreover, some GABAreceptor genes that are related to EEG patterns are also associated with the risk to develop alcohol dependence. These associations again suggest that genetic factors play a major role in the EEG differences associated with alcohol dependence.

The EEG coherence analysis is a technique that investigates the pairwise correlations of power spectra obtained from different electrodes. It measures the functional interaction between cortical areas in different frequency bands. A high level of coherence between two EEG signals indicates a co-activation of neuronal populations and provides information on functional coupling between these areas (Franken et al. 2004). De Bruin et al. (2004, 2006) investigated the pure effects of alcohol intake on synchronization of brain activity, while minimizing the confounding influence of genetic factors related to alcohol dependence. They showed that heavily drinking students with a negative family history had stronger EEG synchronization at theta and gamma frequencies than lightly drinking students with a negative family history. This study suggests that, in students, heavy alcohol intake has an impact on functional brain activity, even in the absence of genetic factors related to alcohol dependence.

The findings of studies on the effects of alcohol dependence on EEG coherence can be summarized as follows: Kaplan et al. (1985) reported lower frontal alpha and slow-beta coherence in alcohol-dependent males and females. Michael et al. (1993) found higher central alpha and slow-beta coherence, but lower parietal alpha and slow-beta coherence in males with alcohol dependence. Winterer et al. (2003a, b) described higher left-temporal alpha and slow-beta coherence and higher slow-beta coherence at right-temporal and frontal electrode pairs in alcohol-dependent males and females. De Bruin et al (2006) showed that moderate-to-heavy alcohol consumption is associated with differences in synchronization of brain activity during rest and mental rehearsal. Heavy drinkers displayed a loss of hemispheric asymmetry of EEG synchronization in the alpha and slow-beta band. Moderately and heavily drinking males additionally showed lower fast-beta band synchronization.

Therefore, qEEG alterations have been described extensively in alcoholics. Most EEG reports in alcoholic patients agree in describing alterations mainly within the beta and alpha bands. Patients with a more pronounced frontal hyperarousal have worse prognosis. Decreased power in slow bands in alcoholic patients may be an indicator of chronic brain damage, while increase in beta band may be related to various factors suggesting cortical hyperexcitability. Abnormalities in resting EEG are highly heritable traits and are often associated with a predisposition to alcoholism development. The studies on the effects of alcohol dependence on EEG coherence can be summarized as lower frontal alpha and slow-beta coherence in alcohol-dependent patients with some topographical coherence abnormality differences between alcoholdependent males and females.

\section{EEG in Marijuana Abuse}

Several lines of evidence suggest that cannabis (marijuana, tetrahydrocannabinol-THC) may alter functionality of the prefrontal cortex and thereby elicit impairments across several domains of complex cognitive function (Egerton et al. 2006). Several studies in both humans and animals have shown that cannabinoid exposure results in alterations in prefrontal cortical activity (Block et al. 2002; O'Leary et al. 2002; Whitlow et al. 2002), providing evidence that cannabinoid administration may affect the functionality of this brain area. Despite the fact that a number of transient physiological, perceptual and cognitive effects are known to accompany acute chronic marijuana (THC) exposure in humans, persistent qEEG effects in humans resulting from continuing exposure to this drug have been difficult to demonstrate (Wert and Raulin 1986). In early reviews of EEG and ERP studies of acute and chronic THC exposure in humans (Struve et al. 1989, 1994), it was reported that significant associations between chronic exposure and clinically abnormal EEG patterns had not been demonstrated and that attempts to use visual EEG analyses to detect transient acute THC exposure induced EEG 
alterations failed to demonstrate consistent THC-EEG effects across studies.

Quantitative methods of analyzing EEG spectra from single posterior scalp derivations began to be applied to studies of acute THC exposure. These early studies reported that acute THC exposure produced transient increases in either posterior alpha power, decreases in mean alpha frequency or increases in alpha synchrony (Fink et al. 1976; Struve et al. 1989; Tassinari et al. 1976; Volavka et al. 1971, 1973). These studies found that THC produced a transient dose-dependent rapid onset: (1) increase in relative power (amount, abundance) of alpha; (2) decrease in alpha frequency; and (3) decrease in relative power of beta as measured from posterior scalp electrodes.

Later studies of Struve et al. (1998, 1999, 2003) demonstrated and replicated a significant association between chronic marijuana use and topographic qEEG patterns of persistent "alpha hyperfrontality" (i.e., elevations of alpha absolute power, relative power, and interhemispheric coherence over frontal cortex) as well as reductions of alpha mean frequency. These findings from chronic users are consistent with both non-topographic (Hockman et al. 1971; Tassinari et al. 1976; Volavka et al. 1973) and topographic (Lukas et al. 1995; Struve et al. 1994) transient EEG effects of acute THC administration. Therefore, chronic daily THC use was found to be associated with distinct topographic qEEG features. Compared with nonusers, THC users had significant elevations of absolute and relative power, and interhemispheric coherence of alpha activity over the bilateral frontal cortex (referred to as "alpha hyperfrontality"). A second finding was that the voltage (not relative power or coherence) of all non-alpha frequency bands was significantly elevated in THC users, although the voltage increase was generalized and not frontally dominant. A third finding involved a widespread decrease in the relative power of delta and beta activity for cannabis users, particularly over the frontal cortical regions. A fourth finding was that interhemispheric coherence of theta and possibly delta activity was also significantly elevated over frontal cortex for marijuana users. Because most studies included daily THC users and non-users drawn from an inpatient psychiatric population, the effects of psychiatric diagnoses or medication were not controlled.

Thus, qEEG studies on acute THC exposure reported a transient dose-dependent increase in relative power of alpha, decrease in alpha frequency, and decrease in relative power of beta at posterior EEG recording sites. Chronic marijuana abuse is known to result in a number of physiological, perceptual and cognitive effects, but persistent qEEG effects from continuing exposure to THC have been difficult to demonstrate. However, recent studies of Struve and his colleagues have demonstrated a significant association between chronic marijuana use and topographic qEEG patterns of persistent elevations of alpha absolute power, relative power, and interhemispheric coherence over frontal cortex, as well as reductions of alpha mean frequency. Another important qEEG finding was the elevated voltage of all non-alpha bands in THC users. A third qEEG finding involved a widespread decrease in the relative power of delta and beta activity over the frontal cortical regions in marijuana users.

\section{EEG in Heroin Addiction}

Only a few studies have investigated qEEG changes in heroin addicts. Qualitative changes were observed in more than $70 \%$ of heroin addicts in the early abstinence (acute withdrawal) period, and these included low-voltage background activity with diminution of alpha rhythm, an increase in beta activity, and a large amount of lowamplitude delta and theta waves in central regions (Olivennes et al. 1983; Polunina and Davydov 2004). Franken et al. (2004) found that abstinent heroin-dependent subjects have an enhanced fast beta power compared with healthy controls, and this finding is concordant with other EEG studies on alcohol and cocaine abusing subjects (Costa and Bauer 1997; Herning et al. 1994b; Rangaswamy et al. 2004; Roemer et al. 1995). Spectral power and eventrelated potentials (ERP) in heroin addicts strongly relate to abstinence length (Shufman et al. 1996, Bauer 2001a; Polunina and Davydov 2004). Most studies showed considerable or even complete normalization of EEG spectral power or magnitude of ERP components in heroin ex-addicts who maintained abstinence for at least 3 months (Bauer 2001b, 2002; Costa and Bauer 1997; Papageorgiou et al. 2001; Polunina and Davidov 2004; Shufman et al. 1996).

Some quantitative changes were also reported in methadone-maintenance heroin addicts (Gritz et al. 1975), current heroin addicts, and subjects in heroin abstinence less than 80 days (Shufman et al. 1996). Gritz et al. (1975) demonstrated a significant slowing of occipital alpha rhythm peak frequency in 10 methadone-maintained patients and the same trend in 10 abstinent heroin-addicted subjects. In one study (Polunina and Davydov 2004), slowing of slow alpha $(8-10 \mathrm{~Hz})$ mean frequency was significantly related to the amount of heroin taken by these patients daily before withdrawal. The prolongation of ERP component latencies in heroin addicts was also reported (Papageorgiou et al. 2001), and these delays significantly correlated with years of heroin use, rather than with abstinence length in the study of Bauer (1997). Polunina and Davydov (2004) demonstrated frequency shifts in the fast alpha range at the frontal and central recording sites 
and a slowing of slow alpha mean frequency at the central, temporal, and occipital sites of recording in heroin abusers who used heroin for at least 18 months.

In general, pronounced desynchronization is characteristic for acute heroin withdrawal, but as it was mentioned above, several studies (Bauer 2001a, 2002; Costa and Bauer 1997; Papageorgiou et al. 2001; Polunina and Davydov 2004; Shufman et al. 1996) showed that spectral power of EEG tends to normalize almost completely after several weeks of abstinence. The most consistent changes in EEG of heroin addicts were reported in alpha and beta frequencies, and included a deficit in alpha activity and an excess of fast beta activity in early heroin abstinence. The latter abnormality appears to reverse considerably when heroin intake is stopped for several months, and therefore it may be viewed as an acute withdrawal effect. The dynamics and characteristics of spectral power changes within the early opiate withdrawal suggest the participation of catecholamine imbalances, especially noradrenaline and perhaps to a lesser degree dopamine, which are widely recognized as a main cause of opiate physical dependency symptoms (Devoto et al. 2002; Maldonado 1997). Acute opiate administration has been shown to increase, while abstinence from chronic opiate use has been shown to decrease extracellular dopamine (DA) in the nucleus accumbens. In contrast, extracellular DA in the prefrontal cortex is not modified by acute opiate use, but is markedly increased during morphine and heroin abstinence syndrome (Devoto et al. 2002). Relationships between theta and beta frequencies shifts and neurotransmitter imbalances characteristic for heroin withdrawal remain unclear.

Withdrawal state in heroin addicts is known to elicit a strong craving for drug, anxiety, nervousness, deficits in inhibitory control, dysphoric motivational state, and intrusive thoughts related to drugs (Franken 2003; Franken et al. 1999, 2004; Stormark et al. 2000). Research on functional connectivity in drug withdrawal states is restricted to a few studies on coherence of the EEG signal in abstinent heroin users (Franken et al. 2004; Fingelkurts et al. (2006a), active heroin abusers (Fingelkurts et al. 2006b), and in abstinent polysubstance abusers (Roemer et al. 1995). In a study on 22 opioid-dependent patients under acute opioid influence, Fingelkurts et al. (2006b) showed that longitudinal opioid exposure impairs cortical local and remote functional connectivity, and found that local connectivity increased, whereas the remote one decreased. These findings were interpreted as specific signs of independent processing in the cortex of chronic heroin addicts. It has been suggested that such independent processes may constitute the candidate mechanism for a welldocumented pattern of impairment in addicts that expresses the lack of integration of different cognitive functions for effective problem solving and helps to explain the observed deficits in abstract concept formation, behavioral control, and problems in the regulation of affect and behavior.

Specifically, Fingelkurts et al. (2006b) found that the number and strength of remote functional connections among different cortical areas estimated by the index of EEG synchrony was significantly higher in patients in acute heroin withdrawal than in healthy controls for most categories of functional connections. Although this result was observed in the alpha as well as in the beta frequency bands, it was most prominent for the beta range. In the same patient sub-sample under acute opioid influence the authors (Fingelkurts et al. 2006a) observed the opposite: a significant decrease in the number and strength of remote functional connections, when compared with healthy controls. Thus, the increase of remote synchronicity among cortical areas during the short-term withdrawal period may indicate the selective attentional focus on cues and memories related to drugs while ignoring neutral cues (Franken et al. 2000; Sokhadze et al. 2007). Generally this can explain a narrowing of the behavioral repertoire and compulsive drug seeking in abstinent addicted subjects (Vanderschuren and Everitt 2004). Therefore, the elevated synchrony within the beta frequency band in these studies (Fingelkurts et al. 2006a, b) may reflect a state of CNS activation toward reward-seeking behavior, with this being a prerequisite of relapse among opiate drug dependent patients (Bauer 2001a).

qEEG changes in heroin addicts in the acute withdrawal period have been described as low-voltage background activity with a diminution of alpha rhythm, an increase in beta activity, and a large amount of low-amplitude delta and theta waves in central regions. In general, pronounced desynchronization is characteristic for acute heroin withdrawal, but the spectral power of EEG tends to normalize almost completely after several weeks of abstinence. The most consistent changes in EEG of heroin addicts were reported in the alpha and beta frequencies, and included a deficit in alpha activity and an excess of fast beta activity in early heroin abstinence. The excess of beta appears to reverse considerably when heroin intake is stopped for several months, and therefore it may be viewed as an acute withdrawal effect. Recent studies found that the number and strength of remote functional connections among different cortical areas estimated by the index of EEG synchrony for the beta range was significantly higher in patients in acute heroin withdrawal than in healthy controls for most categories of functional connections.

\section{EEG in Cocaine Addiction}

Qualitative and quantitative EEG measures are highly sensitive to the acute and chronic effects of neurointoxication 
produced by such psychostimulants as cocaine, as well as effects from withdrawal and long-term abstinence from cocaine use (Ehlers et al. 1989). However, some EEG characteristics observed in cocaine addicts are considered to be due to the toxic effects of this drug on the brain, whereas some EEG characteristics in cocaine addicts may also indicate a predisposition toward the development of SUD (Porjesz et al. 2005).

Hans Berger (1937, cited by Gloor 1969; Herning et al. 1985) was the first to study the effects of cocaine on human EEG, reporting an increase in activity in the beta bandwidth. This was replicated in subsequent studies with a larger number of subjects (Alper 1999; Alper et al. 1990, 1998; Costa and Bauer 1997; Herning et al. 1985; Noldy et al. 1994; Prichep et al. 1996, 1999, 2002; Roemer et al. 1995). Beside beta effects, studies have reported an increase in delta activity (Herning et al. 1985) and frontal alpha activity (Herning et al. 1994b), while others have reported an increase in alpha wave EEG associated with bursts of cocaine-induced euphoria (Lukas 1991). More recently, researchers have begun analyzing qEEG profiles of cocaine-dependent patients using the spectral power of each primary bandwidth over the different topographic cortical areas. Excess alpha activity (Alper et al. 1990; Herning et al. 1994b; Lukas 1991; Prichep et al. 1996) and decreased delta activity (Alper et al. 1990; Noldy et al. 1994; Prichep et al. 1996; Roemer et al. 1995) have been reported, while others have reported increased beta power (Herning et al. 1985, 1994b; Noldy et al. 1994) in cocainedependent patients, recorded in eyes closed, resting conditions. The qEEG abnormalities, primarily found in anterior cortical regions, were shown to correlate with the amount of prior cocaine use (Herning et al. 1996a; Prichep et al. 1996; Roemer et al. 1995; Venneman et al. 2006). The qEEG has been used more often to characterize the effects of withdrawal in cocaine-dependent patients. Several studies reported that during protracted abstinence from cocaine qEEG effects are featured by long-lasting increases in alpha and beta bands together with reduced activity in delta and theta bands (Alper et al. 1990; Prichep et al. 1996; Roemer et al. 1995).

Recently Reid et al. (2006) investigated qEEG profiles in cocaine-dependent patients in response to an acute, single-blind, self-administered dose of smoked cocaine base $(50 \mathrm{mg})$ versus placebo. Cocaine produced a rapid increase in absolute theta, alpha, and beta power over the prefrontal cortex, lasting up to $25 \mathrm{~min}$ after administration of the drug. The increase in theta power was correlated with a positive subjective drug effect ("high"), and the increase in alpha power was correlated with nervousness. Cocaine also produced a similar increase in delta coherence over the prefrontal cortex, which was correlated with nervousness. Placebo resulted only in a slight increase in alpha power over the prefrontal cortex. These data demonstrate the involvement of the prefrontal cortex in the qEEG response to acute cocaine, and indicate that slow wave qEEG, delta and theta activity are involved in the processes related to experiencing rewarding properties of cocaine.

Prichep et al. (1999, 2002) extended the idea of relating baseline EEG activity to outcome in cocaine-dependent patients in treatment programs. Subjects with cocaine dependence have persistent changes in brain function assessed with qEEG methods, present when evaluated at baseline, 5-14 days after last reported crack cocaine use, and persistent at one and six month follow-up evaluations (Alper 1999; Alper et al. 1990, 1998; Prichep et al. 1996, 2002; Venneman et al. 2006). Several recent studies employing qEEG techniques have already demonstrated an association between the amount of beta activity in the spontaneous EEG and relapse in cocaine abuse (Bauer 1997, 2001a). A decrease in the delta and theta bands of the EEG can be regarded as a specific sign of brain dysfunction.

However, this sign, as well as other qEEG abnormal patterns, can be found in many different psychiatric disorders and none of them can be considered as pathognomonic of any specific mental or neurological disorder. EEG coherence in cocaine addiction was investigated in only one study (Roemer et al. 1995). The authors reported globally reduced interhemispheric coherence in the delta and theta bands, and frontally in the beta band. It should be noted that subjects in this study were cocainepreferring polysubstance abusers during abstinence and these results can hardly be generalized to crack cocaineonly users or other categories of cocaine-dependent subjects not enrolled in any treatment.

Therefore, acute effects of smoked crack cocaine have been shown to produce a rapid increase in absolute theta, alpha, and beta power over the prefrontal cortex, lasting up to half-an-hour after administration of the drug. The increase in theta power was reported to correlate with a positive subjective drug effect, while the increase in alpha power was reported to correlate with nervousness. qEEG measures are also sensitive to the acute and chronic effects of cocaine, as well as the effects from withdrawal and longterm abstinence from cocaine use. Some EEG characteristics observed in cocaine addicts are considered to be due to the neurotoxic effects, whereas some EEG characteristics in cocaine addicts may also indicate a predisposition toward the development of cocaine addiction. qEEG has been used more often to characterize the effects of withdrawal in cocaine-dependent patients. During protracted abstinence from cocaine qEEG effects are featured by long-lasting increases in alpha and beta bands together with reduced activity in delta and theta bands. Several recent studies employing qEEG techniques have demonstrated an 
association between the amount of beta activity in the spontaneous EEG and relapse in cocaine abuse.

\section{EEG in Methamphetamine Addiction}

Several studies have examined the neurobiological consequences of methamphetamine dependence using qEEG methods (e.g., Newton et al. 2003, 2004). It was found that methamphetamine dependent patients exhibited a significant power increase in the delta and theta bands as compared to non-drug-using controls (Newton et al. 2003). These results are in accordance with other neurocognitive studies (Kalechstein et al. 2003) suggesting that methamphetamine abuse is associated with psychomotor slowing and frontal executive deficits. Within the methamphetamine-dependent subjects, increased theta qEEG power was found to correlate with response time and was accompanied with reduced accuracy (Newton et al. 2004). To our knowledge, qEEG patterns associated with acute withdrawal and recent abstinence in methamphetamine dependence have not yet been sufficiently described. One study reported (Newton et al. 2003) that methamphetamine dependent volunteers with 4 days of abstinence had increased EEG power in the delta and theta but not in the alpha and beta bands. Within the methamphetamine dependent group, a majority of the conventional EEGs were abnormal $(64 \%)$, compared to $18 \%$ in the nonmethamphetamine using group.

The qEEG may provide a sensitive neurophysiological outcome measure of methamphetamine abuse-related persistent alterations in neurocognitive functions (Newton et al. 2004). In a study by Simon et al. (2002), when performance of patients with SUD was compared to their matched non-using control groups, both methamphetamine and cocaine abusers were impaired on cognitive measures, but the type and degree of impairments were somewhat different. Some of these differences between methamphetamine and cocaine effects on cognitive functions and electrophysiological alterations can be explained by differential pharmacokinetics of these two drugs, as cocaine is rapidly metabolized with an elimination half-life of several hours, whereas methamphetamine is eliminated more slowly, with an elimination half-life averaging $12 \mathrm{~h}$ (Cook et al. 1993; Jeffcoat et al. 1989). Moreover, cocaine differs from methamphetamine in that cocaine inhibits the reuptake of dopamine, serotonin, and norepinephrine, whereas methamphetamine mobilizes and releases these monoamines from storage granules, thus producing rapid and large increases in synaptic concentrations (Simon et al. 2002, 2004). This might be responsible for the discrepancies in observed qEEG manifestations associated with chronic methamphetamine and cocaine abuse.
Only a few studies have examined the qEEG consequences of methamphetamine dependence. They report that methamphetamine dependent patients exhibited a significant power increase in the delta and theta bands as compared to non-drug-using control. The qEEG patterns associated with acute withdrawal and recent abstinence in methamphetamine dependence have not yet been sufficiently described. One study reported that abstinent methamphetamine dependent patients had increased EEG power in the delta and theta but not in the alpha and beta bands. In general, qEEG studies of methamphetamine addiction are in accordance with other neurocognitive studies suggesting that methamphetamine abuse is associated with psychomotor slowing and frontal executive deficits.

\section{P300 Abnormalities in Cocaine, Methamphetamine, Heroin Addiction, and Alcoholism}

The P300 component of the ERP, occurring 300-600 ms post-stimulus, is the most widely used ERP in psychiatry and other clinical applications (Polich et al. 1994; Polich and Herbst 2000; Pritchard 1981, 1986; Pritchard et al. 2004). The amplitude of the P300 reflects the allocation of attentional resources, while the latency is considered to reflect stimulus evaluation and classification time (Katayama and Polich 1998; Polich and Herbst 2000). The P300 is usually obtained in an oddball paradigm, wherein two stimuli are presented in a random order, one of them frequent (standard) and another one rare (target) (Polich 1990). A modification of the oddball task has been used where a third, also rare stimulus (distracter), is presented along with standard and target stimuli. It was reported that these infrequent distracters elicit a frontocentral P300, so called P3a, whereas the rare targets elicit a parietal P300, so called P3b (Katayama and Polich 1996, 1998). The P3a is recorded at the anterior scalp locations and has been interpreted as reflecting frontal lobe activity (Gaeta et al. 2003; Knight 1984). Though the P300 response in general is thought to represent "context updating/closure," in a three-stimuli oddball task the P3a is interpreted as "orienting," and the $\mathrm{P} 3 \mathrm{~b}$ is viewed as an index of the ability to maintain sustained attention to target (Näätänen 1990). The anterior P3a indexes the contextual salience of the rare stimuli, whereas the posterior P3b is indexing task-relevance of the stimuli (Gaeta et al. 2003).

A robust finding in ERP studies on alcoholism is that alcoholics as well as individuals at high risk to develop alcoholism have been shown to have a low P300 amplitude in various task paradigms (Cohen et al. 2002; Hada et al. 2000; Porjesz et al. 2005; Porjesz and Begleiter 1998). Kouri et al. (1996) examined the P300 component in 
patients who were dually dependent on cocaine and heroin. The results showed no P300 amplitude differences between the patients and healthy non-drug-dependent volunteers when patients presented for detoxification. However, after the course of detoxification, the P300 amplitude was significantly smaller in the cocaine- and heroin-dependent group than in the non-dependent control group. In a study by Bauer (2001b) the P300 did not differentiate among patients characterized by histories of either cocaine, or cocaine and alcohol, or heroin dependence. Across all the patient groups, the P300 was significantly reduced in amplitude relative to the P300 ERPs recorded from individuals with no history of alcohol or drug dependence. This study also demonstrated that continued abstinence from heroin and from cocaine and alcohol is also associated with a trend toward normalization of the P300. In a recent study of Papageorgiou et al. (2004) the P300 component was evaluated during the anticipatory period of a short memory task in 20 patients characterized by a past history of heroin dependence (6 months abstinence), in 18 current heroin users and in 20 matched healthy subjects. Abstinent heroin addicts exhibited a significant reduction of the P300 amplitude at the central frontal region, relative to the other two groups.

The results of early work examining the effect of cannabis use and THC administration on visual and auditory ERPs have been inconclusive (Rodin et al. 1970; Roth et al. 1973). Later studies of Patrick et al. (1995, 1997) could not find P300 latency differences in audio and visual oddball tasks between THC users without psychiatric problems and controls. Although THC users displayed reduced auditory and visual P300 amplitudes in this study, when age differences between THC users and controls were removed, all significant P300 amplitude differences were removed as well.

Acute and chronic use of cocaine exerts neuropharmacological effects on amplitude and latency of both anterior and posterior P300 ERP components (Biggins et al. 1997; Fein et al. 1996; Herning et al. 1994a; Kouri et al. 1996; Polich 1990). Longer P300 (P3b) latency without abnormalities in amplitude was reported in several studies on cocaine withdrawal (Herning et al. 1994a; Lukas 1993). Noldy and Carlen (1997) demonstrated effects of cocaine withdrawal on the latency of the P300 in an auditory oddball task. In cocaine-dependent patients, P3a amplitude decrements over frontal areas are persistent even after long periods of abstinence (Bauer 1997). The latency of the P3a was delayed and the amplitude was reduced to novel nontargets in cocaine and alcohol-dependent subjects compared to controls (Biggins et al. 1997; Hada et al. 2000) in auditory and visual three-stimuli oddball tasks.

Several studies have investigated ERP changes associated with methamphetamine abuse and dependence. The
P300 component of the auditory ERP was reported to show a prolonged latency in the oddball task in methamphetamine dependent subjects with a history of psychosis, compared to normal controls (Iwanami et al. 1994, 1998). In particular, the patients with methamphetamine dependence showed reduced P3a amplitude in the reading task and delayed P3b latency with normal P3b amplitude in the auditory oddball task. This was interpreted as indicating a prolonged central noradrenergic dysfunction due to earlier methamphetamine use.

In most ERP studies the P300 did not differentiate among patients characterized by histories of either cocaine, or cocaine and alcohol, or heroin dependence. Across all the patient groups, the P300 was significantly reduced in amplitude relative to P300 ERPs recorded from individuals with no history of alcohol or drug dependence. The latency of the frontal and parietal P300 was reported to be delayed, and the amplitude was reduced to novel non-targets in cocaine and alcohol-dependent subjects compared to controls in auditory and visual three-stimuli oddball tasks. Continued abstinence from heroin, cocaine, and alcohol was shown to be associated with a trend toward P300 normalization. Several studies have investigated ERP changes associated with methamphetamine abuse and dependence. In general, chronic psychoactive substance abuse and drug dependence are associated with delayed and attenuated cognitive ERP in auditory and visual oddball tasks.

qEEG and ERP Abnormalities in Addiction:

Psychopharmacological Effects or Trait Markers?

Whether qEEG alterations and P300 decrements found in most of SUD are only a coincident "marker" of vulnerability or make a direct etiologic contribution to risk for substance dependence is still unknown (Bauer and Hesselbrok 2001; Carlson et al. 2002; O'Connor et al. 1994; Polich et al. 1994; Porjesz and Begleiter 1998). The P300 reduction and abnormal qEEG patterns are seen in mental disorders that often are comorbid with substance abuse, such as conduct disorder (Bauer and Hesselbrock 1999, 2001), ADHD (Bauer 1997; O'Connor et al. 1994), and bipolar or major affective disorder (Friedman and Squires-Wheeler 1994). Reduced P300 amplitude related to prefrontal brain dysfunction may suggest that a deficit in inhibitory control is an underlying mechanism shared by different psychopathologies (Bauer and Hesselbrock 1999; Clark et al. 1999; Tarter et al. 2003). According to Bauer (2002), certain ERP and qEEG abnormalities and impaired functioning on complex cognitive tests in patients formerly dependent on cocaine might not be proximately caused by drug use per se but be more related to comorbid alcohol use 
or another psychiatric condition. Taken together, the findings converge on the conclusion that there exists an inherited predisposition for an externalizing psychopathology that includes ADHD, conduct disorder, and substance abuse. PTSD seems to heighten the risk for addiction as well. Thus, the reviewed findings support the hypothesis that addicted subjects may manifest a P300 amplitude reduction and qEEG abnormalities as a trait reflecting the CNS disinhibition, which may be a predisposing factor for addiction liability, resistance to drug habit extinction, and relapse vulnerability.

\section{Heritability and Neurotransmitter Considerations in Substance Use Disorders}

There has been a consistent drift in addiction research between the psychosocial, cognitive and behavioral aspects of addiction and the biological and genetic emphasis. In much of the present data relating to genetics and animal models (Blum et al. 2006; Porjesz et al. 2005; Ryabinin and Weitemier 2006; Samochowiec et al. 2006), studies suggest that a genetic predisposition for SUD is an accepted concept. Much of the genetic research addresses the influence of alleles thought responsible in coding for genes that express phenotypic neurotransmitter production and distribution; mainly involving endorphins, dopamine and serotonin. These neurotransmitters, dopamine in particular, are also suspect in other appetitive and mood disorders and psychopathologies, of particular note, Reward Deprivation Syndrome (RDS). RDS is described as a dysfunction in the Brain Reward Cascade and proposes that abnormal craving behavior is a consequence of defects in the DRD2 and D1, D3, D4 and D5 dopaminergic receptor genes (Blum et al. 2006).

Blum and colleagues $(1990,1993,1996)$ described this syndrome and identified the D2 dopamine receptor gene as a possible candidate for susceptibility to alcoholism in severe alcoholics (Blum et al. 1993) and proposed this gene's association with dopamine production and distribution may produce a sevenfold increase in the likelihood of developing alcohol use problems (Uhl et al. 1993). This DRD2 dopamine receptor gene and polymorphisms within its genetic coding specific to addiction remain unclear due to its involvement in other disorders; including, obesity (Blum et al. 2006), Tourette's syndrome (Comings et al. 1991) pathological aggression and violence, PTSD (Comings et al. 1996) and schizoid-avoidant disorder (Chen et al. 2005). SUD were classified as a subtype of RDS and treatment regimens for these disorders have been classified as inadequate (Blum et al. 2007) and research continues in developing possible genetic interventions that may produce dopamine and other neurotransmitter regulation in substance-induced rapid dopamine increase in limbic regions (Blum et al. 2007).

It is clear that heritability plays an important role in addictive disorders, however, to what extent environment, perception and synaptic permanency and plasticity influence the course of genetic adaptation or maladaptive traits requires further investigation. Suggested neuroanatomical substrates involved in SUD implicate mesolimbic and diencephalon regions; including the substantia nigra, reticular formation, medial forebrain bundle, nucleus accumbens, septum pediculum, olfactory tubercule and hippocampus and suggest that any concentration of alcohol exposure to these regions would make alcohol use virtually unavoidable (Myers and Privette 1989).

\section{Studies of EEG Biofeedback in Substance Abuse Treatment}

The Peniston Protocol (Alpha-Theta Feedback)

The early studies of Kamiya (e.g., Nowlis and Kamiya 1970) on self-regulation of alpha rhythm elicited substantial interest in the potential clinical applications of alpha biofeedback for SUD treatment. There were reported several uncontrolled case studies and conceptual reviews on alpha EEG training for alcohol (DeGood and Valle 1978; Denney et al. 1991; Jones and Holmes 1976; Passini et al. 1977; Tarbox 1983; Watson et al. 1978) and drug abuse treatment (Brinkman 1978; Goldberg et al. 1976, 1977; Lamontagne et al. 1977; Sim 1976), but the impact of alpha biofeedback training as a SUD therapy was not significant.

The bulk of the literature to date regarding EEG biofeedback of addictive disorders is focused on alpha-theta biofeedback. The technique involves the simultaneous measurement of occipital alpha $(8-13 \mathrm{~Hz})$ and theta $(4$ $8 \mathrm{~Hz}$ ) and feedback by separate auditory tones for each frequency representing amplitudes greater than pre set thresholds. The subject is encouraged to relax and to increase the amount of time the signal is heard, that is to say, to increase the amount of time that the amplitude of each defined bandwidth exceeds the threshold. A variety of equipment and software has been used to acquire, process, and filter these signals, and there are differences in technique inherent with equipment and software.

Alpha-theta feedback training was first employed and described by Elmer Green and colleagues (Green et al. 1974) at the Menninger Clinic. This method was based on Green's observations of single lead EEG during meditative states in practiced meditators, during which increased theta amplitude was observed following an initial increased alpha amplitude, then a drop off of alpha amplitude (theta/ 
alpha crossover). When the feedback of the alpha and theta signal was applied to subjects, states of profound relaxation and reverie were reported to occur. The method was seen as useful in augmenting psychotherapy and promoting individual insight. It could be seen as a use of brain wave signal feedback to enable a subject to maintain a particular state of consciousness similar to a meditative or hypnotic relaxed state over a 30 - or 40-min feedback session.

Goslinga (1975) gave the first description of the use of alpha-theta feedback in a SUD treatment program. This integrated program started in 1973 at the Topeka VA, and included group and individual therapies. Daily 20-min EEG biofeedback sessions (integrated with EMG biofeedback and temperature control biofeedback) were conducted over 6 weeks, resulting in free, loose associations, heightened sensitivity, and increased suggestibility. Patients discussed their insights and experiences associated with biofeedback in therapy groups several times a week, augmenting expressive psychotherapy. The first published clinical reports of efficacy of alpha-theta training at the Topeka VA were by Twemlow and Bowen (1976), who explored the impact of alpha-theta training on psychodynamic issues in 67 non-psychotic chronic male alcoholics in an inpatient treatment program. In this non-controlled study, they found that "religiousness" as a predictor of "self-actualization" may have increased as a result of imagery experienced in theta states. This was seen as positive to the program goal of augmenting Alcoholics Anonymous as a recovery philosophy. The high suggestibility of the method was acknowledged; "treatments such as brainwave training, which utilize abstract, ill understood techniques are potential repositories of magical projection and fantasy and would logically be more acceptable to alcoholics who are able to have 'faith' (devoutly or moderately religious)" (Twemlow and Bowen 1977). In another uncontrolled study at the Topeka VA, 21 alcoholics were reported to exhibit within and across session increases in raw theta amplitudes at occipital areas bilaterally measured by single lead EEG during the course of alpha-theta training, becoming more able to achieve deep states as manifested by EEG (Twemlow et al. 1977). These initial studies advanced the utility of biofeedback induced theta states in promoting insight and attitude change in alcoholics, with the assumptions that biofeedback-induced theta states are associated with heightened awareness and suggestibility, and that this heightened awareness and suggestibility would enhance recovery. Outcome data regarding abstinence were not reported.

In the first reported randomized and controlled study of alcoholics treated with alpha-theta EEG biofeedback, Peniston and Kulkosky (1989) described positive outcome results. Their subjects were inpatients in a VA hospital treatment program, all males with established chronic alcoholism and multiple past failed treatments. Following a temperature biofeedback pre-training phase, Peniston's experimental subjects $(n=10)$ completed 15 30-min sessions of eyes closed occipital alpha-theta biofeedback. Compared to a traditionally treated alcoholic control group $(n=10)$, and nonalcoholic controls $(n=10)$, alcoholics receiving brainwave biofeedback showed significant increases in percentages of EEG recorded in the alpha and theta rhythms, and increased alpha rhythm amplitudes (single lead measurements at international site O1). The experimentally treated subjects showed reductions in Beck Depression Inventory scores compared to the control groups. Control subjects who received standard treatment alone showed increased levels of circulating beta-endorphin, an index of stress, whereas the EEG biofeedback group did not. Thirteen-month follow-up data indicated significantly more sustained prevention of relapse in alcoholics who completed alpha-theta brainwave training as compared to the control alcoholics, defining successful relapse prevention as "not using alcohol for more than six contiguous days" during the follow-up period. In a further report on the same control and experimental subjects, Peniston and Kulkosky (1990) described substantial changes in personality test results in the experimental group as compared to the controls. The experimental group showed improvement in psychological adjustment on 13 scales of the Millon Clinical Multiaxial Inventory compared to the traditionally treated alcoholics who improved on only two scales and became worse on one scale. On the 16-PF personality inventory, the neurofeedback training group demonstrated improvement on seven scales, compared to only one scale among the traditional treatment group. This small $\mathrm{n}$ study employed controls and blind outcome evaluation, with actual outcome figures of $80 \%$ positive outcome versus $20 \%$ in the traditional treatment control condition at 4-year follow up.

The protocol described by Peniston at the Fort Lyons VA cited above is similar to that initially employed by Twemlow and colleagues at the Topeka VA and Elmer Green at the Menninger Clinic, with two additions, i.e., (1) temperature training and (2) script. Peniston introduced temperature biofeedback training as a preconditioning relaxation exercise, along with an induction script to be read at the start of each session. This protocol (described as follows) has become known as the "Peniston Protocol" and has become the focus of research in subsequent studies. Subjects are first taught deep relaxation by skin temperature biofeedback for a minimum of five sessions that additionally incorporates autogenic phrases. Peniston also used the criteria of obtaining a temperature of $94^{\circ}$ before moving on to EEG biofeedback. Participants then are instructed in EEG biofeedback and in an eyes closed and relaxed condition, receive auditory signals from an EEG 
apparatus using an international site O1 single electrode. A standard induction script employing suggestions to relax and "sink down" into reverie is read. When alpha (8-12 Hz) brainwaves exceed a preset threshold, a pleasant tone is heard, and by learning to voluntarily produce this tone, the subject becomes progressively relaxed. When theta brainwaves $(4-8 \mathrm{~Hz})$ are produced at a sufficiently high amplitude, a second tone is heard, and the subject becomes more relaxed and according to Peniston, enters a hypnagogic state of free reverie and high suggestibility. (Although theta increase and alpha decrease are thought by Peniston to be associated with a deeply relaxed state where hypnagogic reverie is present, this may simply represent drowsiness) (Niedermeyer 1999). Following the session, with the subject in a relaxed and suggestible state, a therapy session is conducted between the subject and therapist where the contents of the imagery experienced is explored and "abreactive" experiences are explored (Peniston and Kulkosky 1989, 1990, 1991).

Saxby and Peniston (1995) reported on 14 chronically alcohol dependent and depressed outpatients using this same protocol of alpha-theta brainwave biofeedback. Following treatment, subjects showed substantial decreases in depression and psychopathology as measured by standard instruments. Twenty-one month follow-up data indicated sustained abstinence from alcohol confirmed by collateral report. These male and female outpatients received 20 40-min sessions of feedback.

Bodenhamer-Davis and Calloway (2004) reported a clinical trial with 16 chemically dependent outpatients, 10 of whom were probationers classified as high risk for re-arrest. Subjects completed an average of 31 alphatheta biofeedback sessions. Psychometrics demonstrated improvements in personality and mood. Follow-up at 74-98 months indicated $81.3 \%$ of the treatment subjects were abstinent. Re-arrest rates and probation revocations for the probation treatment group were lower than those for a probation comparison group (40\% vs. 79\%).

Fahrion (1995) gave a preliminary report $(n=119)$ on a large randomized study of alpha-theta training for addiction in the Kansas Prison System using group-training equipment. A report of the completed study $(n=520)$ (Fahrion 2002) showed little difference between the two groups overall at 2-year outcome. But, when results were analyzed for age, race and drug of choice, neurofeedback emerged as a more efficacious treatment for younger and non-white and non-stimulant abusing participants. Interestingly, this protocol was not effective for cocaine abusers. (Stimulant abusers will be discussed later in this article under the Scott-Kaiser modification of the Peniston protocol.)

The issue of alpha-theta biofeedback in culturally sensitive groups that have not responded to traditional modes of addiction treatment (such as confrontational group therapies) has been considered in an open case series reported by Kelly (1997). This three year follow-up study presented the treatment outcomes of 19 Dine' (Navajo) clients. Four $(21 \%)$ participants achieved "sustained full remission," $12(63 \%)$ achieved "sustained partial remission," and $3(16 \%)$ remained "dependent." The majority of participants also showed a significant increase in "level of functioning".

Schneider et al. (1993) used slow cortical potential biofeedback to treat 10 unmedicated alcoholic patients in four neurofeedback sessions after hospitalization. Seven patients participated in a fifth session an average of 4 months later. Six out of these seven patients had not had a relapse at the follow-up. These results are similar to those reported for alpha theta training.

Several other studies using the Peniston protocol and its modifications reported cases with positive clinical effects (Burkett et al. 2003, DeBeus et al. 2002; Fahrion et al. 1992; Finkelberg et al. 1996; Skok et al. 1997). These studies suggest that an applied psychophysiological approach based on an alpha-theta biofeedback protocol is a valuable alternative to conventional substance abuse treatment (Walters 1998). Nevertheless, most of these results were reported at the society meetings, and only few of these studies were published in mainstream peerreviewed journals other than The Journal of Neurotherapy.

A critical analysis of the Peniston Protocol is discussed at length in the previous reviews (Trudeau 2000, 2005a, b). Several controlled studies of the Peniston protocol for addictions, completed by Lowe (1999), Moore and Trudeau (1998), and Taub and Rosenfeld (1994), suggest that alphatheta training for addictions may be non-specific in terms of effect when compared to suggestion, sham or controlled treatment, or meditational techniques. By contrast, Egner et al. (2002) showed that alpha-theta training results in an increase of theta/alpha ratios, as compared to a control condition. In an in depth critical analysis that examines inconsistencies reported in the original Peniston papers, Graap and Freides (1998) raise serious issues about the reporting of original samples and procedures in these studies. In their analyses, the results may have been due as much to the intense therapies accompanying the biofeedback as due to the biofeedback itself. The subjects may have been comorbid for a number of conditions, which were not clearly reported, particularly PTSD, which may have been the focus of the treatment. In his reply to these criticisms, Peniston (1998) acknowledges that it "remains unknown whether the temperature training, the visualizations, the ATBWNT (alpha-theta brain wave neurotherapy), the therapist, the placebo, or the Hawthorne effects are responsible for the beneficial results." The criticism raised above by Graap and Friedes (1998) 
regarding Peniston's papers could also be applied to earlier replication studies. Neither Peniston's studies nor the replication studies provide sufficient detail regarding the specifics of the types of equipment used for alpha-theta feedback, including filtering methods for the EEG signal or other technical information, to permit exact reproduction of the feedback protocols with other equipment. Outcome criteria also vary in the replication studies, with varying measures of abstinence and improvement. An exception to these concerns is the report of Scott et al. (2005), which will be discussed later in greater detail.

It should be noted that psychostimulant (cocaine, methamphetamine) addictions may require approaches and neurofeedback protocols other than alpha/theta training. Persons who are cocaine-dependent are cortically underaroused during protracted abstinence (Roemer et al. 1995). qEEG changes, such as a decrease in high beta (18-26 Hz) power are typical for withdrawal from cocaine (Noldy et al. 1994). Cocaine abusers who are still taking this drug often show low amounts of delta and excess amounts of alpha and beta activity (Alper 1999; Prichep et al. 1999), whereas chronic methamphetamine abusers usually exhibit excessive delta and theta activity (Newton et al. 2003). Thus, cocaine and methamphetamine users may warrant a different EEG biofeedback protocol, at least at the beginning stages of neurofeedback therapy.

\section{The Scott-Kaiser Modification of the Peniston Protocol}

Scott and Kaiser (1998) describe combining a protocol for attentional training (beta and/or SMR augmentation with theta suppression) with the Peniston protocol (alpha-theta training) in a population of subjects with mixed substance abuse, rich in stimulant abusers. The beta protocol is similar to that used in ADHD (Kaiser and Othmer 2000) and was used until measures of attention normalized, and then the standard Peniston protocol without temperature training was applied (Scott et al. 2002). The study group is substantially different than that reported in either the Peniston or replication studies. The rationale is based in part on reports of substantial alteration of qEEG seen in stimulant abusers associated with early treatment failure (Prichep et al. 1996, 2002) likely associated with marked frontal neurotoxicity and alterations in dopamine receptor mechanisms (Alper 1999). Additionally, preexisting ADHD is associated with stimulant preference in adult substance abusers, and is independent of stimulant associated qEEG changes. These findings of chronic EEG abnormality and high incidence of preexisting ADHD in stimulant abusers suggest they may be less able to engage in the hypnagogic and auto-suggestive Peniston protocol (Trudeau et al. 1999). Furthermore, eyes-closed alpha feedback as a starting protocol may be deleterious in stimulant abusers because the most common EEG abnormality in crack cocaine addicts is excess frontal alpha (Prichep et al. 2002).

In their initial report, Scott and Kaiser (1998) described substantial improvement in measures of attention and also of personality (similar to those reported by Peniston and Kulkosky 1990). Their experimental subjects underwent an average of 13 SMR-beta $(12-18 \mathrm{~Hz})$ neurofeedback training sessions followed by 30 alpha-theta sessions during the first 45 days of treatment. Treatment retention was significantly better in the EEG biofeedback group and was associated with the initial SMR-beta training. A subsequent published paper (Scott et al. 2005) reported on an expanded series of 121 inpatient drug program subjects randomized to condition, followed up at 1 year. Subjects were tested and controlled for the presence of attentional and cognitive deficits, personality states and traits. The experimental group showed normalization of attentional variables following the SMR-Beta portion of the neurofeedback, while the control group showed no improvement. Experimental subjects demonstrated significant changes $(p<.05)$ beyond the control subjects on 5 of the 10 scales of the MMPI-2. Subjects in the experimental group were also more likely to stay in treatment longer and more likely to complete treatment as compared to the control group. Finally, the one-year sustained abstinence levels were significantly higher for the experimental group as compared to the control group.

The approach of beta training in conjunction with alphatheta training has been applied successfully in a treatment program aimed at homeless crack cocaine abusers in Houston, as reported by Burkett et al. (2003), with impressive results. Two hundred and seventy (270) male addicts received 30 sessions of a protocol similar to the Scott Kaiser modification. One-year follow-up evaluations of 94 treatment completers indicated that $95.7 \%$ of subjects were maintaining a regular residence; $93.6 \%$ were employed/in school or training, and $88.3 \%$ had no subsequent arrests. Self-report depression scores dropped by $50 \%$ and self-report anxiety scores by $66 \%$. Furthermore, $53.2 \%$ reported no alcohol or drug use 12 months after biofeedback, and $23.4 \%$ used drugs or alcohol only one to three times after their stay. This was a substantial improvement from the expected $30 \%$ or less expected recovery in this group. The remaining $23.4 \%$ reported using drugs or alcohol more than 20 times over the year. Urinalysis results corroborated self-reports of drug use. The treatment program saw substantial changes in length of stay and completion. After the introduction of the neurofeedback to the mission regimen, length of stay tripled, beginning at 30 days on average and culminating at 100 days after the addition of neurotherapy. In a later study 
the authors reported follow-up results on 87 subjects after completion of neurofeedback training (Burkett et al. 2005). The follow-up measures of drug screens, length of residence, and self-reported depression scores showed significant improvement. It should be noted that this study had limitations, because neurofeedback was positioned only as an adjunct therapy to all other faith-based treatments for crack cocaine abusing homeless persons enrolled in this residential shelter mission and was an uncontrolled study. Yet the improvement in program retention is impressive and may well be related to the improved outcome.

\section{Continuing Research}

\section{Self-Perception and Experimental Schemata in the Addicted Brain}

Rex Cannon, Joel Lubar, and Deborah Baldwin of the Brain Research and Neuropsychology Laboratory at University of Tennessee at Knoxville are performing research with three goals in mind: First, to attempt to reconcile and integrate data from all disciplines involved in addiction research in order to develop a novel approach for neurophysiological study pertaining to SUD and conceivably determine and describe EEG source generators that are instrumental in the processes of self-perception and experiential schemata utilizing a recently developed assessment instrument. Second, to utilize this information to develop an integrative treatment model for addictive disorders based on this research, involving novel group processing methods and spatial specific neurophysiological operant learning (LORETA Neurofeedback) (Cannon et al. 2006, 2007; Congedo 2003; Congedo et al. 2004), and finally, third, to utilize both the assessment and neurophysiological data for development of statistical models for possible diagnostic and predictive purposes and to provide a means for a neurophysiological measure of treatment efficacy.

Research indicates that substance abusers have elevated beta activity in an EEG resting state as compared with normative groups (Rangaswany et al. 2002) and elevated alpha activity after administering a mood altering substance (Cohen et al. 1993; Kaplan et al. 1985). It is suggested that many of the neurophysiological markers may provide information about the state of the individual prior to the development of an addictive disorder and that these brain functions are under genetic control (Porjesz et al. 2002, 2005; Tapert 2004). Kaplan et al. (1985) reported lower frontal alpha and slow-beta coherence in alcohol-dependent males and females. Michael et al. (1993) found higher central alpha and slow-beta coherence, but lower parietal alpha and slow-beta coherence in males with alcohol dependence; contrarily, other findings suggest that morphine, alcohol and marijuana show increased alpha 2 power in the spectral EEG and relate this to the euphoric state produced by the drug (Lukas 1989, 1995). Winterer et al. (2003a, b) described higher left-temporal alpha and slow-beta coherence and higher slow-beta coherence at right-temporal and frontal electrode pairs in alcoholdependent males and females. De Bruin et al. (2004) showed that moderate-to-heavy alcohol consumption is associated with differences in synchronization of brain activity during rest and mental rehearsal. Heavy drinkers displayed a loss of hemispheric asymmetry of EEG synchronization in the alpha and low-beta band. Moderately and heavily drinking males additionally showed lower fastbeta band synchronization. Decision-making processes and the ability to form a resistance to drugs, i.e., the ability to say no, involve numerous brain regions; including, the insular, somatosensory, orbitofrontal, anterior cingulate and dorsolateral prefrontal cortices, as well as the amygdala, hippocampus and thalamic nuclei (Bechara 2005).

This research considers the integration of the features of addicted persons as reported in earlier studies, case reports and theoretical concepts as vital in understanding behavioral manifestations of the suspected neural pathways that are premised to be involved in the development of SUD. Some of the fundamental descriptions of addicted individuals portray them as passive with dependent strivings, emotionally immature, abounding with fears of responsibility or independent action and ultimately, infantile inadequate personalities (Coodley 1961), as well as emotionally, socially, and educationally underdeveloped (Meyerstein 1964), and immature and regressive (Dorsey 1961; Gerard and Kornetsky 1955; Hill 1962). These individuals are reported to struggle with affirming positive thoughts of self-esteem, tendencies to undervalue themselves and be self deprecating, and exhibit difficulty adjusting to others and these tendencies are veiled by overt behavioral patterns, including, physical or verbal abuse.

Individuals with SUD present with a vast number of paradoxical characteristics; including an overwhelming sense of inadequacy disguised by an apparent overwhelming sense of confidence. Similarly, an apparent abundance of anger and aggression utilized as a disguise for a paralyzing sense of fear, more specifically, fear of people, economic insecurity, rejection, and alienation, which paradoxically are exacerbated by the continued use of the substance. One of the more profound idiosyncratic characteristics of this population is the tendency to ruminate and associate past events, perceptions and the associated emotions with both present and future. The perception of experience is often clouded by the personalization of events (real or imagined) and reinforced with a deliberate, ambiguous effort to avoid reconciling this 
confound, which reinforces an uninhibited association of all current interactions and situations with past events. Opposite to what often is implied, these features may not originate from the consequences of substance abuse, but from earlier periods in development (Vos 1989), and in the perspective of this research these features and others have an etiology in specific neurophysiological regions that are the direct result of dendritic pruning that occurs in early development that continues on into adolescence and, unless intervention or awareness of these schemata are achieved, they remain problematic into adulthood.

To date, studies identifying such schematic source generators and their relationship with SUD using qEEG and standardized low-resolution electromagnetic tomography (sLORETA) are scant. This research is designed to assess the neural activation patterns relative to schemata regarding the self in recovering addicts and identify possible generators in the cortex as compared to controls. In this research, it is hypothesized that there is dendritic pruning early in developmental phases that contribute to frequency specific activity in neuronal populations in the ventromedial portions of the prefrontal cortex and limbic regions. Furthermore, it is proposed that these neural pathways hinder the integration of affect, cognition, reward and decision-making processes and adversely influence the perception of self and self in relation to experience and the development of adaptive schemata and personality characteristics.

Integration of Cognitive Neuroscience Approaches in Assessment of Functional Outcomes of Neurofeedback and Behavioral Therapy Based Interventions in SUD

Sokhadze et al. (2007a) in their conceptual review proposed an integrated approach to assessment and treatment utilizing cognitive neuroscience methods (e.g., qEEG, ERP), conventional psychotherapeutic treatment, and neurofeedback therapy to assess the recovery of cognitive and emotional functions affected by chronic psychostimulant drug abuse co-occurring with PTSD. Cognitive neuroscience methodologies used for assessment of the outcome effects of psychotherapy and neurofeedback interventions for comorbid disorders have significant potential for additionally identifying neurophysiological and clinical markers of treatment progress (Sokhadze 2005). These outcome markers may provide useful information for planning bio-behavioral interventions in this form of dual diagnosis.

Stotts and colleagues (2006) at the University of Texas at Houston, in collaboration with researchers at Rice University, used motivational interviewing (MI) with personalized feedback, particularly employing the ERP markers of deficiencies in selective attention task produced by cocaine abuse in crack addicts. In a randomized, controlled pilot study these authors (Stotts et al. 2006; Sokhadze et al. 2004, 2005) evaluated the feasibility and preliminary efficacy of a brief MI intervention using EEG/ERP graphical feedback for crack cocaine abusers. Treatment-seeking cocaine abusers $(N=31)$ were randomly assigned to a two-session MI intervention or a general educational drug conseiling (control) condition. All participants received EEG assessments based on dense-array ERP tests in a selective attention task at intake and post-treatment. Results indicated that the MI intervention was feasible and the subjective impact of the EEG/ERP feedback was positive. Significant group differences in percentage of cocaine positive urine screens across the study were found, favoring the MI group; $84.9 \%$ for the control group and $62.6 \%$ in the MI group.

In a current study at the University of Louisville, Tato Sokhadze and his colleagues are utilizing dense-array qEEG/ERP variables and measures of behavioral performance on mental tasks (reaction time, accuracy) to explore the cognitive functions in patients with cocaine abuse/ dependence diagnosis, and the recovery of these functions during bio-behavioral intervention based on an integrated neurofeedback approach (NFB, Scott-Kaiser protocol) and motivational enhancement therapy (MET) in an outpatient population. The purpose of this research is also to characterize changes in cognitive functioning associated with the success rate of three arms for cocaine addiction treatment (MET, NFB, combined MET + NFB). Prior, during, and subsequent to the above bio-behavioral therapies, individual differences in qEEG and dense-array ERP are being assessed during cognitive tasks containing drug-related and generally affective cues, and during cognitive tasks aimed to test cortical inhibitory capacity, selective attention, response error processing, and cortical functional connectivity. Preliminary data from this study were presented at the 2007 annual meeting of ISNR (Sokhadze et al. 2007b) and are being prepared for the publication.

\section{Efficacy of Alpha Theta Training}

The Guidelines for Evaluation of Clinical Efficacy of Psychophysiological Interventions (LaVaque et al. 2002), which have been accepted by AAPB and ISNR, specify five types of classification for the effectiveness of biofeedback procedures, ranging from "Not empirically supported" to "Efficacious and Specific." The requirements for each 
classification level are summarized in brief below. A more complete description may be found in LaVaque et al. (2002).

\section{Criteria for Levels of Evidence of Efficacy}

Level 1: Not empirically supported. This classification is assigned to those treatments that have only been described and supported by anecdotal reports and/or case studies in non-peer reviewed journals.

Level 2: Possibly efficacious. This classification is considered appropriate for those treatments that have been investigated in at least one study that had sufficient statistical power, well-identified outcome measures, but lacked randomized assignment to a control condition internal to the study.

Level 3: Probably efficacious. Treatment approaches that have been evaluated and shown to produce beneficial effects in multiple observational studies, clinical studies, wait list control studies, and within-subject and betweensubject replication studies merit this classification.

Level 4: Efficacious. In order to be considered "efficacious," a treatment must meet the following criteria:

(a) In a comparison with a no-treatment control group, alternative treatment group, or sham (placebo) control utilizing randomized assignment, the investigational treatment is shown to be statistically significantly superior to the control condition or the investigational treatment is equivalent to a treatment of established efficacy in a study with sufficient power to detect moderate differences;

(b) The studies have been conducted with a population treated for a specific problem, from whom inclusion criteria are delineated in a reliable, operationally defined manner;

(c) The study used valid and clearly specified outcome measures related to the problem being treated;

(d) The data are subjected to appropriate data analysis;

(e) The diagnostic and treatment variables and procedures are clearly defined in a manner that permits replication of the study by independent researchers, and

(f) The superiority or equivalence of the investigational treatment have been shown in at least two independent studies" (LaVaque et al. 2002, p. 280).

Level 5: Efficacious and Specific. To meet the criteria for this classification, the treatment needs to be demonstrated to be statistically superior to a credible sham therapy, pill, or bona fide treatment in at least two independent studies.
Using these criteria and based on the studies reported to date alpha-theta training can be classified as Level 3probably efficacious-when combined with an inpatient rehabilitative treatment modality in subjects with long standing alcohol dependency. This classification is based on the original randomized and controlled study of the Peniston Protocol (Peniston and Kulkosky 1989, 1990, 1991) and multiple observational and uncontrolled studies that preceeded (Twemlow and Bowen 1977, Twemlow et al. 1977) and followed these studies (DeBeus et al. 2002; Burkett et al. 2003; Fahrion et al. 1992; Finkelberg et al. 1996; Skok et al. 1997; Bodenhamer-Davis and Calloway 2004; Saxby and Peniston 1995; Fahrion 1995).

Using these criteria and based on reported studies to date the Scott-Kaiser modification of the Peniston Protocol can also be classified as probably efficacious (Level 3) when combined with residential rehabilitation modalities in stimulant abusers. This rating is based on one controlled study of 121 subjects in which Peniston's outcomes of both psychometric improvement and abstinence improvement were replicated (Scott et al. 2005) and one observational study of 71 subjects (Burkett et al. 2003).

Alpha-theta training protocols do not completely meet the criteria for the Level 4, "efficacious" classification. Although there are sufficient studies that show statistically significant superiority of randomly assigned treatment groups to no-treatment control groups, and studies have been conducted with populations treated for a specific problem, from whom inclusion criteria are delineated in a reliable, operationally defined manner, and the studies cited use valid and clearly specified outcome measures related to the problem being treated with data subjected to appropriate data analysis, there remains the shortcoming cited by Graap and Freides (1998) for the initial reports of Peniston and Kulkosky $(1989,1990,1991)$. We recall the qualifying limitations of LaVaque et al. (2002), who stated that "the diagnostic and treatment variables and procedures are not clearly defined in a manner that permits replication of the study by independent researchers" (p. 280). However, the Scott et al. (2005) report does appear to clearly delineate treatment variables and procedures. One other independent study showing the superiority of modified alpha-theta training to control condition would meet the stated criteria for a Level 4 "efficaceous" classification.

To be considered Level 5 ("efficacious and specific") modified alpha-theta training would need to be shown to be superior to sham or bona fide treatment. It has not been demonstrated that the Peniston type alpha-theta feedback is more efficacious than sham treatment (Trudeau 2000, 2005a, b; Lowe 1999; Moore and Trudeau 1998) or alternative treatment that involves meditation (Taub and Rosenfeld 1994). 


\section{Clinical Considerations: Comorbidities of SUD and Implications for Individualized (qEEG-Guided) Neurofeedback}

There are several conditions commonly associated with addictive disorders that have known neurophysiological aberrations. The co-occurrence of alcohol and other SUD with other psychiatric disorders has been widely recognized. Co-occurrence of SUD and other psychiatric diagnosis (e.g., PTSD, antisocial personality disorder, ADHD, unipolar depression etc.) is highly prevalent (Drake and Walach 2000; Evans and Sullivan 1995; Grant et al. 2004; Jacobsen et al. 2001). Persons with co-occurring other mental disorders and SUD have a more persistent illness course and are more refractive to treatment than those without dual diagnoses (Brown et al. 1995; O'Brien et al. 2004; Schubiner et al. 2000; Swartz and Lurigio 1999). Depression occurs in approximately $30 \%$ of chronic alcoholics (Regier et al. 1990). In treatment settings, these depressed patients can present particular challenges to the clinician, as they may not respond as well to treatment as other patients, may have greater relapse, attrition, and readmission rates, and may manifest symptoms that are more severe, chronic, and refractory in nature (Sheehan 1993). Independent of other psychiatric comorbidity, ADHD alone significantly increases the risk for SUD (Biederman et al. 1995). Associated social and behavioral problems may make individuals with comorbid SUD and ADHD treatment resistant (Wilens et al. 1998). In males ages 16-23, the presence of childhood ADHD and conduct disorder is associated with non-alcohol SUD (Gittleman et al. 1985; Manuzza et al. 1989). In summary childhood ADHD associated with conduct disorder in males is an antecedent for adult non-alcohol SUD and antisocial personality disorder (Wender 1995). The incidence of ADHD in clinical SUD populations has been studied and may be as high as 50\% for adults (Downey et al. 1997) and adolescents (Horner and Scheibe 1997). Adult residual ADHD is especially associated with cocaine abuse and other stimulant abuse (Levin and Kleber 1995). Monastra et al. (2005) in a white paper review of ADHD, cite positive treatment outcomes of just under $80 \%$ in treatment of ADHD with neurofeedback.

Rates of PTSD occurring in persons primarily identified with or in treatment for substance abuse vary from $43 \%$ (Breslau et al. 1991) up to 59\% (Triffleman et al. 1999). In a general population study, Cottler et al. (1992) reported that cocaine abusers were three times more likely to meet diagnostic criteria for PTSD compared to individuals without a SUD. Kalechstein et al. (2000) found that methamphetamine-dependent individuals are at greater risk to experience particular psychiatric symptoms. There was reported a significant dependence-by-gender effect, with methamphetamine-dependent females reporting significantly more overall posttraumatic stress symptomatology compared to females reporting no dependence, whereas males significantly differed only with respect to depression. Peniston and Kulkosky (1991) reported effective treatment of PTSD using a protocol similar to the one they employed for alcoholics.

Hughes and John (1999) review the applicability of qEEG findings in SUD. They note that in numerous qEEG studies there is a consensus of increased beta relative power in alcoholism and increased alpha in cannabis and crack cocaine users. They conclude that the evidence provided by studies to date is insufficient to recommend qEEG as a routine clinical assessment tool in SUD, although it may be useful in differential diagnosis in difficult cases. A number of specific qEEG abnormalities have been described as specific to suspected neurotoxicities associated with chronic stimulant abuse. These studies (Alper et al. 1990; Noldy et al. 1994; Prichep et al. 1996; Roemer et al. 1995; Trudeau et al. 1999) based on reasonably uniform abstinence times and employing different EEG technology and analytical approaches, have produced remarkably similar findings of alpha relative amplitude excess with delta relative amplitude deficit that is striking. Excess alpha amplitude with slowing of alpha frequency associated with chronic cannabis abuse has been reported (Struve et al. 1998). As noted, Scott and Kaiser (1998) describe combining a protocol for attentional training (beta reward) with alpha-theta training in a population of subjects whose primary drugs of abuse were stimulants and who had features of ADHD.

It may make good sense clinically to consider specific neurotherapy treatment of these disorders either in place of or preceding alpha-theta therapy, similar to the ScottKaiser approach. Second, applicable neurotherapy approaches are attractive alternative therapies for coexisting or underlying conditions in SUD clients who have high-risk behaviors for medication treatment, such as overdosing, abuse, or poor compliance. While there are no published systematic studies of neurotherapy treatment of co-occurring depression, TBI, ADHD, PTSD, or drug neurotoxicity on the course and outcome of addictive disorders, several recent reports of neurotherapy for addictions based on qEEG findings, which in turn may be related to comorbidities, have been presented. Basically, this technique involves the use of qEEG to identify patterns of EEG that deviate from standardized norms, and individualized EEG biofeedback protocols to correct them (Romano-Micha 2003). DeBeus et al. (2002) are presently conducting a randomized controlled study of neurotherapy for SUD that examines the difference between a qEEGbased treatment, a research-based (Scott-Peniston) treatment, and a wait-list control for chemically dependent 
outpatients. Preliminary results are promising. While historically, alpha-theta training has been the accepted approach in treating chemical dependency, this study suggests qEEG-based training is a viable alternative, demonstrating similar outcomes for personality change and abstinence rates. Future directions include determination of those likely to benefit from one of the particular treatments or a combination of the two and analysis of long-term abstinence rates. Gurnee (2004) has presented data on a series of 100 sequential participants with SUD who were treated by qEEG-based neurotherapy, with marked heterogeneity of qEEG subtypes and corresponding symptom complexes. In this clinically derived scheme, qEEGs that deviate from normative databases, mainly with excess alpha amplitude, are associated more often with depression and ADD. Those with deficient alpha amplitude are associated with anxiety, insomnia, and alcohol/drug abuse. Beta excess amplitude is associated with anxiety, insomnia, and alcohol/drug abuse. Central abnormalities are interpreted as mesial frontal dysfunction and are associated with anxiety, rumination, and obsessive compulsive symptoms. The therapeutic approach is to base neurotherapy on correcting identified qEEG abnormalities, i.e., train beta excess amplitude down when present, while monitoring symptoms.

Tentative findings suggest that qEEG variables may be used to predict those alcoholics and drug abusers most at risk for relapse. Winterer et al. (1998) were able to predict relapse among chronic alcoholics with $83-85 \%$ success, significantly outperforming prediction from clinical variables. Although they found more desynchronized (less alpha and theta and more beta activity) over frontal areas in alcoholics in general, those individuals who relapsed displayed even more of this activity. Bauer (2001) obtained EEG data on alcohol, cocaine or opioid dependent patients after 1-5 months of sobriety. Those who had relapsed by 6 months later were also characterized by increased beta $(19.5-39.8 \mathrm{~Hz})$ activity relative to those maintaining abstinence. Relative beta power was superior to severity of the alcoholism, depression level, antisocial personality disorder, childhood conduct problems, family history, or age as predictors, and was unaffected by the substance of abuse. The EEG differences between relapse-prone and abstinence-prone groups were found to be related to the interaction of two premorbid factors: childhood conduct disorder and paternal alcoholism. These findings receive further support from Bauer (1993) and from Prichep et al. (1996) who also found that beta activity was predictive of treatment failure. They found two clusters among cocaine addicts: One had more severe damage (alpha) and tended to remain in treatment. Those with less severe alpha excess and more beta activity tended to leave treatment. They also discovered that dropouts could not be determined from the presence of anxiety or depression or demographic variables.

Treatment of patients with substance abuse disorder by neurofeedback may become more complicated when patients present various psychiatric conditions. When addiction is comorbid with ADHD it is suggested that SMR (or beta increase, theta decrease) training should be conducted to address the related ADHD disorder first (Biederman et al. 1997). Applicability of neurofeedback methods to treat anxiety and affective disorders is reviewed by Hammond (2006). Peniston and Kulkosky (1990) describe personality normalization in alcoholics treated with EEG biofeedback. Alpha-theta feedback has also been reported as efficacious in alcoholics with depressive symptoms (Saxby and Peniston 1995). There are only a few case studies on the efficacy of neurofeedback for treating generalized anxiety disorder (Vanathy et al. 1998) and PTSD (Huang-Storms et al. 2006; Graap et al. 1997). Alpha-theta feedback has been described as efficacious in post-combat PTSD (Peniston and Kulkosky 1991; Peniston et al. 1993). However, additional research needs to be completed to determine the clinical outcome and efficacy of bio-behavioral treatment based on brain wave self-regulation in addiction disorders that are comorbid with various anxiety disorders and PTSD.

\section{Clinical Considerations: Cognitive-Behavioral and Neurofeedback Treatment in Substance Use Disorders}

Because of its chronic nature, long-term treatment for SUD is usually necessary (Crits-Christoph et al. 1997, 1999). Effective agonist and antagonist pharmacotherapies as well as symptomatic treatments exist for opioid dependence, but neither agonists nor antagonists have been approved as uniquely effective for treatment of stimulant abuse or dependence (Grabowski et al. 2004). There is no current evidence supporting the clinical use of carbamazepine (Tegretol), antidepressants, dopamine agonists (drugs commonly used to treat Parkinson's and Restless Leg Syndrome), disulfiram (Antabuse), mazindol (an experimental anorectic), phenytoin (Dilantin), nimodipine (Nimotop), lithium and other pharmacological agents in the treatment of cocaine dependence (de Lima et al. 2002; Venneman et al. 2006). Because no proven effective pharmacological interventions are available for cocaine addiction or for methamphetamine addiction, treatment of stimulant addiction has to rely on existing cognitivebehavioral therapies (CBT) or CBT combined with other biobehavioral approaches (Van den Brink and van Ree 2003).

According to Volkow et al. (2004) successful strategies for behavioral treatment in drug addiction may include (1) 
interventions aimed to decrease the reward value of the drug and simultaneously increase values of natural reinforcement; (2) approaches aimed to change stereotype conditioned drug-seeking behaviors; and (3) methods to train and strengthen frontal inhibitory control. Because stressful events can result in relapse to drug taking behavior (Koob and Le Moal 2001), an adjunct treatment strategy is to interfere with the neurobiological responses to stress (Goeders 2003; Koob and Le Moal 2001). Treatment of comorbid mental conditions may also require the concurrent treatment of drug addiction. In some cases, however, comorbid drug addiction may result from attempts to alleviate the psychiatric disorder through selfmedication (i.e., co-occurring cocaine use and ADHD and/ or heroin addiction co-occurring with PTSD). In other cases, severity of a psychiatric disorder symptom may increase as a result of drug abuse (Volkow et al. 2003, 2004).

In patients with drug abuse arising from an attempt to self-medicate (Khantzian 1985, 1997), treatment of the comorbid mental disorder may help prevent abuse. For instance, treatment of the preexisting condition of ADHD may prevent cocaine abuse (Biederman et al. 1995, 1997). In some cases though the persistent qEEG abnormalities associated with chronic SUD may happen to be independent from ADHD clinical status (Trudeau et al. 1999). The co-occurrence of ADHD and SUD has received considerable attention in the recent clinical and scientific literature (Davids et al. 2005). These two disorders are often linked to one another. Because the core symptoms of ADHD may be mimicked by the effects of psychoactive drugs, it is difficult to diagnose one disorder in the presence of the other (Davids et al. 2005). ADHD has been found to be associated with an earlier onset of SUD (Horner and Scheibe 1997). It is generally assumed that untreated ADHD is a risk factor for SUD development (Biederman et al. 1997, 1998; Manuzza et al. 1998; Trudeau 2005a, b).

In a case of comorbidity in which the use of drugs antecedes a mental disease (e.g., substance-induced anxiety disorder, DSM-IV-TR, APA 2000) or is not driven by selfmedication strategies, the simultaneous treatment of both psychiatric conditions may be required. In this situation, treatment could be guided by the two following concepts: (1) Behavioral interventions to activate and strengthen circuits involved in inhibitory control, such as bio-behavioral self-regulation training, may increase successful abstinence from drug taking. (2) Considering the important role of cognitive and emotional processes involved in the predisposition for drug abuse, the development of nonpharmacological interventions (e.g., CBT, stress management, neurofeedback) is a feasible strategy.

\section{Directions for Further Research}

Specific patterns of qEEG abnormality associated with specific substance use toxicity such as those found in stimulant abuse or alcohol abuse or with comorbidities such as ADHD (Chabot and Serfontein 1996), PTSD (HuangStorms et al. 2006) or TBI (Thatcher et al. 1989) suggest underlying brain pathologies that might be amenable to EEG biofeedback that is tailored to the person. These approaches would likely be individualized rather than protocol based, and would be used independently or in conjunction with classic alpha-theta training. By way of example, these could include protocols specific to the qEEG abnormality, such as frontal delta reward to correct the frontal delta deficit in cocaine abuse that Alper (1999) hypothesizes may be related to cocaine sensitization and changes in dopamine transmission. To our knowledge this has never been studied and is clearly a research (not a clinical) recommendation. The qEEG patterns and abnormalities depend significantly on whether the subject is still currently using, the chronicity of use, and the current stage of withdrawal or protracted abstinence. A neurofeedback protocol selected for an individual client with SUD should be directly related to the level of current substance use or abstinence, especially in such classes of drugs as heroin, where the withdrawal syndrome results in substantial physiological manifestations including transient qEEG changes.

Even though there are no reported systematic studies of EEG biofeedback treatment of commonly occurring comorbidities of SUD, it makes sense that clinical EEG biofeedback treatment study protocols consider the presence of ADHD, TBI, depression, and drug-associated neurotoxicity. This approach may improve outcome, especially in conventional treatment resistant participants.

Unfortunately, only a few large-scale studies of neurofeedback in addictive disorders have been reported in the literature. Most, if not all of the recommendations previously made regarding further research (Trudeau 2000, 2005b) have yet to be implemented. These recommendations are summarized as follows.

(1) Studies require external, systematic replicability of brain wave feedback methods and results in diverse populations that include various control and alternative treatment conditions wherein the groups are matched on key dimensions.

(2) Details need to be given regarding the equipment that was used and the associated technical specifications (e.g., details about amplification, filtering, spectral extraction, windowing, and other pertinent information) needed by neurofeedback specialists for replication and comparison. 
(3) The essential components and durations for brain wave feedback required for therapeutic advantage need to be stated, including double-blinded studies that control for all other possible therapeutic effects.

(4) Open clinical trials that investigate efficacy of the types of protocols used for ADHD, PTSD, depression, and TBI remediation with SUD subjects comorbid for those conditions need to be reported.

(5) Open clinical trials that assess the efficacy of EEG biofeedback in addressing the specific qEEG changes of chronic alcohol, heroin, cannabis and stimulant abuse need to be reported.

(6) The physiological and psychological processes of the therapeutic effects of EEG biofeedback, including studies of qEEG and ERP changes, need to be investigated and reported.

(7) Studies need to adhere to clearly defined outcome measures that have established reliability and validity.

Other important recommendations for future development of the field are listed below:

(a) The availability of an increased number of channels for EEG and ERP recording (e.g., higher spatial sampling rate) makes it possible to better localize the source of brain activity. More focused research of this type seems warranted.

(b) There are several specific functional diagnostic tools from the cognitive neuroscience arsenal that are very specific for testing addictive disorders. Those that may be especially valuable include cue reactivity tests using qEEG and ERP measures. Cue reactivity is a very sensate test of motivational relevance of drugrelated items (Carter and Tiffany 1999) that can be detected using EEG methods.

(c) In addition to using more traditional neurocognitive tests (TOVA, IVA+, etc.) that are commonly included in neurofeedback research (e.g., in particular in studies on effectiveness of neurotherapy in ADHD treatment) there may be value in incorporating standardized tests with EEG/ERP recording to assess executive functions in addicts. Tests that warrant mention are the Continuous Performance Test (GoNoGo task), Stroop test, Eriksen flanker test, etc. Some of these tests are sufficiently sensitive for assessing recovery of cortical inhibition function commonly known to be impaired in patients with SUD.

(d) Testing emotional reactivity and responsiveness in addiction is another important domain where qEEG and ERP methods may help to obtain more effective evaluation of the affective state of recovering addicts.
In future neurofeedback treatment for SUD attempts should be made to integrate neurotherapy with other well known behavioral interventions for drug abuse, such as cognitive-behavioral therapy (CBT) and motivation enhancement therapy (MET, Miller and Rollnick 2002). As a population, drug addicts are very difficult to treat, characterized by a low motivation to change their drug habit and a reluctance to enter inpatient treatment. CBT and MET are powerful psychotherapeutic interventions that can help to bring about rapid commitment to change addictive behaviors. These behavioral therapies are especially useful for enhancing compliance with drug-dependent individuals and facilitating their neurofeedback treatment engagement.

Neurofeedback may be among the most promising biofeedback modalities for the treatment of adolescents with addictive disorders because of the neuroplasticity potential of the adolescent brain. While there is little work available on the prevention and treatment of SUD in adolescents utilizing neurotherapy, there is no reason to suspect that the approaches used in adults would not be applicable in SUD adolescents (Trudeau 2005b).

The EEG biofeedback treatment of ADHD may be important in prevention for children and adolescents at risk for developing SUD. It may be possible that EEG biofeedback therapy of childhood ADHD may result in a decrease in later life SUD (Wilens et al. 1998). This remains speculative, as there have been no reported studies of the effects of neurofeedback treatment on prevention of SUD to date.

There are several important applications of the neurofeedback protocols for enhancement of cognitive performance in healthy subjects (reviewed in Vernon 2005). This promising new line of neurofeedback-based cognitive neuroscience research (Barnea et al. 2005; Egner and Gruzelier 2001, 2003, 2004a, b; Egner et al. 2004; Vernon et al. 2003) has significant potential to elucidate neurobiological mechanisms explaining how neurofeedback training may alter and enhance cognition and behavioral performance in patients with SUD as well.

Drugs of abuse can impair cognitive, emotional and motivational processes. More qEEG and cognitive ERP research is needed to characterize the chronic and residual effects of drugs on attention, emotion, memory, and overall behavioral performance. More research is needed also to relate cognitive functionality measures to clinical outcome (e.g., relapse rate, drug screens, psychiatric status, etc.). Such qEEG/ERP studies may facilitate the translation of clinical neurophysiology research data into routine practical tools for assessment of functional recovery both in alcoholism and addiction treatment clinics. We believe that administration of some of above described qEEG assessments at the pre-treatment baseline might provide useful predictors of clinical outcome and relapse risk. 
Incorporation of cognitive tests with EEG and ERP (e.g., P300) measures into cognitive-behavioral and neurofeedback based interventions may have significant potential for identifying whether certain qEEG/ERP measures can be used as psychophysiological markers of treatment progress (and/or relapse vulnerability), and also may provide useful information in planning cognitive-behavioral and neurotherapy treatment when substance abuse is comorbid with a mental disorder.

With the advances made in the last several years, it is hoped that continued interest will be generated to further study brainwave biofeedback treatment of addictive disorders. Effectiveness in certain "hard to treat" populations (conventional treatment resistant alcoholics, crack cocaine addicts, cognitively impaired substance abusers) is promising. The prospect of an effective medication free, neurophysiologic, and self-actualizing treatment for a substance based, brain impaired, and self-defeating disorder such as SUD is attractive.

Acknowledgements This project was partially sponsored by NIDA and ISNR pilot research grants to Tato Sokhadze.

\section{References}

Alper, K. R. (1999). The EEG and cocaine sensitization. Journal of Neuropsychiatry and Clinical Neuroscience, 11, 209-221.

Alper, K. R., Chabot, R. J., Kim, A. H., Prichep, L. S, \& John, E. R. (1990). Quantitative EEG correlates of crack cocaine dependence. Psychiatry Research, 35, 95-106.

Alper, K. R., Prichep, L. S., Kowalik, S., Rosenthal, M. S, \& John, E. R. (1998). Persistent QEEG abnormality in crack cocaine users at 6 months of drug abstinence. Neuropsychopharmacology, 19, $1-9$.

American Psychiatric Association. (2000). Diagnostic and statistical manual of mental disorders (4th ed.) [DSM-IV], Text revised. Washington, DC.

Barnea, A., Rassis, A., \& Zaidel, E. (2005). Effect of neurofeedback on hemispheric word recognition. Brain \& Cognition, 59, 314-321.

Bauer, L. O. (1993). Motoric signs of CNS dysfunction associated with alcohol and cocaine withdrawal. Psychiatry Research, 47(1), 69-77.

Bauer, L. O. (1997). Frontal P300 decrement, childhood conduct disorder, family history, and predisposition of relapse among abstinent cocaine abusers. Drug \& Alcohol Dependence, 44, $1-10$.

Bauer, L. O. (2001a). Predicting relapse to alcohol and drug abuse via quantitative electroencephalography. Neuropsychopharmacology, 25, 332-333.

Bauer, L. O. (2001b). CNS recovery from cocaine, cocaine and alcohol, or opioid dependence: A P300 study. Clinical Neurophysiology, 112(8), 1508-1515.

Bauer, L. O. (2002). Differential effects of alcohol, cocaine, and opiod abuse on event-related potentials recorded during a response competition task. Drug \& Alcohol Dependence, 66, 137-145.

Bauer, L. O., \& Hesselbrock, V. M. (1999). P300 decrements in teenagers with conduct problems Implications for substance abuse risk and brain development. Biological Psychiatry, 46, 263-272.

Bauer, L. O., \& Hesselbrock, V. M. (2001). CSD/BEM localization of P300 sources in adolescents "at risk": Evidence of frontal cortex dysfunction in conduct disorder. Biological Psychiatry, 50, 600608.

Bauer, L. O., \& Hesselbrock, V. M. (2002). Lateral asymmetries in the frontal brain: Effects of depression and a family history of alcoholism in female adolescents. Alcoholism Clinical and Experimental Research, 26, 1662-1668.

Bechara, A. (2005). Decision making, impulse control and loss of willpower to resist drugs: A neurocognitive perspective. Nature Neuroscience, 8(11), 1458-1463.

Biederman, J., Wilens, T., Mick, E., Faraone, S. V., \& Spenser, T. (1998). Does attention-deficit hyperactivity disorder impact the developmental course of drug and alcohol abuse and dependence? Biological Psychiatry, 44, 269-273.

Biederman, J., Wilens, T., Mick, E., Faraone, S. V., Weber, W., Curtis, S., Thornell, A., Pfiser, K., Jetton, J. G., \& Soriano, J. (1997). Is ADHD a risk factor for psychoactive substance use disorders? Findings from a four-year prospective follow-up study. Journal American Academy Child Adolescent Psychiatry, $36,21-29$.

Biederman, J., Wilens, T., Mick, E., Milberger, S., Spencer, T. J., \& Farone, S. V. (1995). Psychoactive substance use disorders in adults with attention deficit hyperactivity disorder (ADHD): Effects of ADHD and psychiatric comorbidity. American Journal of Psychiatry, 152(11), 1652-1658.

Biggins, C. A., MacKay, S., Clark, W., \& Fein, G. (1997). Eventrelated potential evidence for frontal cortex effects of chronic cocaine dependence. Biological Psychiatry, 42, 472-485.

Block, R. I., O’Leary, D. S., Hichwa, R. D., Augustinack, J., Boles Ponto, L., Ghoneim, M. M., Arndt, S., Hurtig, R., Watkins, G. L., Hall, J., Nathan, P. E., \& Andreasen, N. C. (2002). Effects of frequent marijuana use on memory-related regional cerebral blood flow. Pharmacology, Biochemistry and Behavior, 72, 237250 .

Blum, K., Chen, T., Meshkin, B., Downs, B., Gordon, C., et al. (2006). Genotrim-A DNA customized nutrigenomic product, targets genetic factors of obesity: Hypothesizing a dopamineglucose correlation demonstrating reward deficiency syndrome (RDS). Medical Hypotheses, 68, 844-852.

Blum, K., Cull, J. G., Braverman, E. R., \& Comings, D. E. (1996). Reward deficiency syndrome. The American Scientist, 84, 132145.

Blum, K., Noble, E. P., Sheridan, P. J., Montgomery, A., Ritchie, T., Jagadeeswaran, P., et al. (1990). Allelic association of human dopamine D2 receptor gene in alcoholism. Journal of American Medical Association, 263, 2055-2060.

Blum, K., Noble, E., Sheridan, P., Montgomery, A., Ritchie, T., et al. (1993). Genetic predisposition in alcoholism: Association of the D 2 dopamine receptor TaqI B 1 RFLP with severe alcoholics. Alcohol, 10, 59-67.

Blum, K., Chen, T., Meshkin, B., Waite, R., Downs, B., et al, (2007). Manipulation of catechol- $O$-methyl-transferase (COMT) activity to influence the attenuation of substance seeking behavior, a subtype of Reward Deficiency Syndrome (RDS), is dependent upon gene polymorphisms: a hypothesis. Medical Hypotheses, 69, 1054-1060.

Bodenhamer-Davis, E., \& Callaway, T. (2004). Extended follow-up of Peniston Protocol results with chemical dependency. Journal of Neurotherapy, 8(2), 135.

Breslau, N., Davis, G. C., Andreski, P., \& Peterson, E. (1991). Traumatic events and posttraumatic stress disorder in an urban population of young adults. Archives of General Psychiatry, 48, 216-222. 
Brinkman, D. N. (1978). Biofeedback application to drug addiction in the University of Colorado drug rehabilitation program. International Journal of Addiction, 13(5), 817-830.

Brown, P. J., Recupero, P. R., \& Stout, R. (1995). PTSD-substance abuse comorbidity and treatment utilization. Addictive Behavior, 20, 251-254.

Burkett, S. V., Cummins, J. M., Dickson, R., \& Skolnick, M. H. (2003). Neurofeedback in the treatment of addiction with a homeless population. Presented at ISNR 11th annual conference, Houston, September 18-21.

Burkett, S. V., Cummins, J. M., Dickson, R., \& Skolnick, M. H. (2005). An open clinical trial utilizing real-time EEG operant conditioning as an adjunctive therapy in the treatment of crack cocaine dependence. Journal of Neurotherapy, 9(2), 27-47.

Cannon, R., Lubar, J., Congedo, M., Thornton, K., Hutchens, T., \& Towler, K. (2007). The effects of Neurofeedback in the cognitive division of the anterior cingulate gyrus. International Journal of Neuroscience, 117(3), 337-357.

Cannon, R., Lubar, J., Gerke, A., Thornton, K., Hutchens, T., \& McCammon, V. (2006). EEG spectral power and coherence: LORETA Neurofeedback in the anterior cingulate gyrus. Journal of Neurotherapy, 10(1), 5-31.

Carlson, S. R., Iacono, W. G., \& McGue, M. (2002). P300 amplitude in adolescent twins discordant and concordant for alcohol use disorder. Biological Psychology, 61, 203-227.

Carter, B. L., \& Tiffany, S. T. (1999). Meta-analysis of cue-reactivity in addiction research. Addiction, 94, 327-340.

Centers for Disease Control Prevention [CDC] (2004). Unintentional and undetermined poisoning deaths-11 states, 1990-2001. Morbidity and Mortality Weekly Reports, 53, 233-238.

Chabot, R. J., \& Serfontein, G. (1996). Quantitative EEG profiles of children with attention deficit disorder. Biological Psychiatry, 40, 951-963.

Chen, T., Blum, K., Mathews, D., Fisher, L., Schnautz, N., et al. (2005). Are dopaminergic genes involved in a predisposition to pathological aggression? Hypothesizing the importance of "super normal controls" in psychiatricgenetic research of complex behavioral disorders. Medical Hypotheses, 65, 703707.

Clark, D., Parker, A., \& Lynch, K. (1999). Psychopathology of substance related problems during early adolescence: A survival analysis. Journal of Clinical Child Psychology, 28, 333-341.

Coodley, A. E. (1961). Current aspects of delinquency and addiction. General Psychiatry, 4, 124-132.

Cohen, H. L., Ji, J., Chorlian, D. B., Begleiter, H., \& Porjesz, B. (2002). Alcohol-related ERP changes recorded from different modalities: A topographic analysis. Alcoholism: Clinical and Experimental Research, 26, 303-317.

Cohen, H., Porjesz, B., \& Begleiter, H. (1993). The effects of ethanol on EEG activity in males at risk for alcoholism. Electroencephalography and Clinical Neurophysiology, 86, 368-376.

Comings, D. E., Comings, B. G., Muhleman, D., Dietz, G., Shahbahrami, B., Tast, D., Knell, E., et al. (1991). The dopamine D2 receptor locus as a modifying gene in neuropsychiatric disorders. Journal of the American Medical Association, 266, 1793-1800.

Comings, D. E., Muhleman, D., \& Gysin, R. (1996). Dopamine D2 receptor (DRD2) gene and susceptibility to posttraumatic stress disorder: A study and replication. Biological Psychiatry, 40, 6872.

Congedo, M. (2003). Tomographic Neurofeedback: A new technique for the Self-Regulation of brain electrical activity. An unpublished dissertation. University of Tennessee, Knoxville.

Congedo, M., Lubar, J., \& Joffe, D. (2004). Low-resolution electromagnetic tomography neurofeedback. IEEE Transactions On
Neuronal Systems and Rehabilitation Engineering, 12(4), 387397.

Cook, C. E., Jeffcoat, A. R., Hill, J. M., Pugh, D. E., Patetta, P. K., Sadler, B. M., White, W. R., \& Perez-Reyes, M. (1993). Pharmacokinetics of methamphetamine self-administered to human subjects by smoking $S$-(+)-methamphetamine hydrochloride. Drug Metabolism Disposition, 21, 717-723.

Costa, L., \& Bauer, L. (1997). Quantitative electroencephalographic differences associated with alcohol, cocaine, heroin and dualsubstance dependence. Drug \& Alcohol Dependence, 46, 87-93.

Cottler, L. B., Compton, W. M., Mager, D., Spitznagel, E. L., \& Janca, A. (1992). Posttraumatic stress disorder among substance users from the general population. American Journal of Psychiatry, 149(5), 664-670.

Coutin-Churchman, P., Moreno, R., Añez, Y., \& Vergara, F. (2006). Clinical correlates of quantitative EEG alterations in alcoholic patients. Clinical Neurophysiology, 117(4), 740-751.

Crits-Christoph, P., Siqueland, L., Blaine, J., Frank, A., Luborsky, L., et al. (1997). The National Institute on Drug Abuse Collaborative Cocaine Treatment Study: Rationale and methods. Archives of General Psychiatry, 54, 721-726.

Crits-Christoph, P., Siqueland, L., Blaine, J., Frank, A., Luborsky, L., Onken, L. S., et al. (1999). Psychosocial treatments for cocaine dependence: National Institute on Drug Abuse Collaborative Cocaine Treatment Study. Archives of General Psychiatry, 56, 493-450.

Dackis, C. A., \& O’Brien, C. P. (2001). Cocaine dependence: A disease of the brain's reward centers. Journal of Substance Abuse Treatment, 21, 111-117.

Darke, S., \& Hall, W. (2003). Heroin overdose: Research and evidence-based intervention. Journal of Urban Health, 80, 189200.

Davids, E., von Bunau, U., Specka, M., Fischer, B., Scherbaum, N., \& Gastpar, M. (2005). History of attention-deficit hyperactivity disorder symptoms and opioid dependence: A controlled study. Progress in Neuro-Psychopharmacology \& Biological Psychiatry, 29, 291-296.

DeBeus, R., Prinzel, H., Ryder-Cook, A., \& Allen, L. (2002). QEEGbased versus research-based EEG biofeedback treatment with chemically dependent outpatients: Preliminary results. Journal of Neurotherapy, 6(1), 64-66.

De Bruin, E. A., Bijl, S., Stam, C. J., Böcker, B. E., Kenemans, J., \& Verbaten, M. N. (2004). Abnormal EEG synchronisation in heavily drinking students. Clinical Neurophysiology, 115, 20482055.

De Bruin, E. A., Stam, C. J., Bijl, S., Verbaten, M. N., \& Kenemans, J. L. (2006). Moderate-to-heavy alcohol intake is associated with differences in synchronization of brain activity during rest and mental rehearsal. International Journal of Psychophysiology, 60(3), 304-314.

DeGood, D. E., \& Valle, R. S. (1978). Self-reported alcohol and nicotine use and the ability to control occipital EEG in a biofeedback situation. Addictive Behaviors, 1, 13-18.

De Lima, M. S., de Oliveira Soares, B. G., Reisser, A. A., \& Farrell, M. (2002). Pharmacological treatment of cocaine dependence: A systematic review. Addiction, 97, 931-949.

Denney, M. R., Baugh, J. L., \& Hardt, H. D. (1991). Sobriety outcome after alcoholism treatment with biofeedback participation: A pilot inpatient study. International Journal of Addiction, 26(3), 335-341.

Dorsey, J. M. (1961). Narcotics addiction in our community primarily an educational problem. The Journal of the Michigan State Medical Society, 60, 621-629.

Devoto, P., Flore, G., Pira, L., Diana, M., \& Gessa, L. (2002). Corelease of noradrenaline and dopamine in the prefrontal cortex 
after acute morphine and during morphine withdrawal. Psychopharmacology, 160(2), 220-224.

Di Chiara, G. (1999). Drug addiction as dopamine-dependent associative learning disorder. European Journal of Pharmacology, 375(1-3), 13-30.

Downey, K. K., Stelson, F. W., Pomerleau, O. F., \& Giordani, B. (1997). Adult attention deficit hyperactivity disorder: Psychological test profiles in a clinical population. Journal of Nervous and Mental Diseases, 185(1), 32-38.

Drake, R. E., \& Wallach, M. A. (2000). Dual diagnosis 15 years of progress. Psychiatry Services, 51, 1126-1129.

Egerton, A., Allison, C., Brett, R. R., \& Pratt, J. A. (2006). Cannabinoids and prefrontal cortical function: Insights from preclinical studies. Neuroscience and Biobehavioral Reviews, 30, 680-695.

Egner, T., \& Gruzelier, J. H. (2001). Learned self-regulation of EEG frequency components affects attention and event-related brain potentials in humans. Neuroreport, 12, 4155-4159.

Egner, T., \& Gruzelier, J. H. (2003). Ecological validity of neurofeedback: Modulation of slow wave EEG enhances musical performance. Neuroreport, 14, 1225-1228.

Egner, T., \& Gruzelier, J. H. (2004a). EEG biofeedback of low beta band components: Frequency-specific effects on variables of attention and event-related brain potentials. Clinical Neurophysiology, 115, 131-139.

Egner, T., \& Gruzelier, J. H. (2004b). The temporal dynamics of electroencephalographic responses to alpha/theta neurofeedback training in healthy subjects. Journal of Neurotherapy, 8, 43-57.

Egner, T., Strawson, E., \& Gruzelier, J. H. (2002). EEG signature and phenomenology of alpha/theta neurofeedback training versus mock feedback. Applied Psychophysiology and Biofeedback, 27(4), 261-270.

Egner, T., Zech, T. F., \& Gruzelier, J. H. (2004). The effects of neurofeedback training on the spectral topography of the electroencephalogram. Clinical Neurophysiology, 115, 2452-2460.

Ehlers, C. L., Wall, T. L., \& Schuckit, M. A. (1989). EEG spectral characteristics following ethanol administration in young men. Electroencephalography \& Clinical Neurophysiology, 73, 179187.

Enoch, M. A., White, K. V., Harris, C. R., Rohrbaugh, J., \& Goldman, D. (2002). The relationship between two intermediate phenotypes for alcoholism: Low voltage alpha EEG and low P300 ERP amplitude. Journal of Studies on Alcohol, 63, 509-517.

Evans, K., \& Sullivan, J. M. (1995). Treating addicted survivors of trauma. New York: Guilford Press.

Fahrion, S. L. (1995). Human potential and personal transformation. Subtle Energies, 6, 55-88.

Fahrion, S. L. (2002). Group biobehavioral treatment of addiction. Paper presented at The 4th Meeting on the Neurobiology of Criminal and Violent Behavior. Research and Clinical Applications of Neurofeedback for Offender Populations with Substance Use Disorders and ADD/ADHD. Federal Bureau of Prisons, Scottsdale AZ, Accessed 26 February.

Fahrion, S. L., Walters, D., Coyne, L., \& Allen, T. (1992). Alterations in EEG amplitude, personality factors and brain electrical mapping after alpha-theta brainwave training: A controlled case study of an alcoholic in recovery. Alcoholism: Clinical Experimental Research, 16, 547-551.

Fein, G., Bigging, C., \& MacKay, S. (1996). Cocaine abusers have reduced auditory P50 amplitude and suppression compared to both normal controls and alcoholics. Biological Psychiatry, 39, 955-965.

Fingelkurts, A. A., Fingelkurts, A. A., Kivisaari, R., Autti, T., Borisov, S., Puuskari, V., Jokela, O., \& Kähkönen, S. (2006a). Reorganization of the composition of brain oscillations and their temporal characteristics in opioid dependent patients. Progress in Neuro-Psychopharmacology and Biological Psychiatry, 30(8), $1453-1465$.

Fingelkurts, A. A., Fingelkurts, A. A., Kivisaari, R., Autti, T., Borisov, S., Puuskari, V., Jokela, O., \& Kähkönen, S. (2006b). Increased local and decreased remote functional connectivity at EEG alpha and beta frequency bands in opioid-dependent patients. Psychopharmacology, 188(1), 42-52.

Fink, M., Volavka, J., Panayiotopoulos, C. P., \& Stefanis, C. (1976). Quantitative EEG studies of marihuana, delta-9-tetrahydrocannabinol and hashish in man. In M. C. Braude \& S. Szara, (Eds.), The pharmacology of marihuana (pp. 383-391). New York: Raven Press.

Finkelberg, A., Sokhadze, E., Lopatin, A., Shubina, O., Kokorina, N., Skok, A., \& Shtark, M. (1996). The application of alpha-theta EEG biofeedback training for psychological improvement in the process of rehabilitation of the patients with pathological addictions. Biofeedback and Self-Regulation, 21, 364.

Finn, P. R., \& Justus, A. (1999). Reduced EEG alpha power in the male and female offspring of alcoholics. Alcoholism: Clinical and Experimental Research, 23(2), 256-262.

Franken, I. H. A. (2003). Drug craving and addiction: Integrating psychological and psychopharmacological approaches. Progress Neuro-Pharmacology Biological Psychiatry, 27, 563-579.

Franken, I. H., de Haan, H. A., van der Meer, C. W., Haffmans, P. M., \& Hendriks, V. M. (1999). Cue reactivity and effects of cue exposure in abstinent posttreatment drug users. Journal of Substance Abuse Treatment, 16, 81-85.

Franken, I. H. A., Kroon, L. Y., \& Hendriks, V. M. (2000). Influence of individual differences in craving and obsessive cocaine thoughts on attentional processes in cocaine abuse patients. Addictive Behaviours, 25, 99-102.

Franken, I. H. A., Stam, C. J., Hendriks, V. M., \& van den Brink, W. (2004). Electroencephalographic power and coherence analysis suggest altered brain function in bstinent male heroin-dependent patients. Neuropsychobiology, 49, 105-110.

French, M. T., McGeary, K. A., Chitwood, D. D., \& McCoy, C. B. (2000). Chronic illicit drug use, health services utilization and the cost of medical care. Social Science \& Medicine, 50, 17031713.

Friedman, D., \& Squires-Wheeler, E. (1994). Event-related potentials (ERPs) as indicators of risk for schizophrenia. Schizophrenia Bulletin, 20(1):63-74.

Gaeta, H., Friedman, D., \& Hunt, G. (2003). Stimulus characteristics and task category dissociate the anterior and posteriror aspects of the novelty P3. Psychophysiology, 40, 198-208.

Gerard, D. L., \& Kornetsky, C. (1955). Adolescent opiate addiction: A study of control and addict subjects. Psychiatric Quarterly, 29, 457-486.

Gerdeman, G. L., Partridge, J. G., Lupica, C. R., \& Lovinger, D. M. (2003). It could be habit forming: Drugs of abuse and striatal synaptic plasticity. TRENDS in Neurosciences, 26(4), 184-192.

Goeders, N. E. (2003). The impact of stress on addiction. European Neuropsychopharmacology, 13, 435-441.

Gurnee, R. (2004). Subtypes of alcoholism and CNS depressant abuse. In Abstracts of the winter brain, optimal functioning, and positive psychology meeting. Palm Springs, 2004 http://www.brainmeeting.com/2004_abstracts.htm.

Gittleman, R., Mannuzza, S., Shenker, R., \& Bonagura, N. (1985). Hyperactive boys almost grown up. Archives of General Psychiatry, 42, 937-947.

Gloor, P. (Ed). (1969). Hans Berger on the electroencephalogram of man. Electroencephalography and Clinical Neurophysiology, Suppl 28, 95-132. Amsterdam: Elsevier.

Goldberg, R. J., Greenwood, J. C., \& Taintor, Z. (1976). Alpha conditioning as an adjunct treatment for drug dependence: Part I. International Journal of Addiction, 11(6), 1085-1089. 
Goldberg, R. J., Greenwood, J. C., \& Taintor, Z. (1977). Alpha conditioning as an adjunct treatment for drug dependence: Part II. International Journal of Addiction, 12(1), 195-204.

Goslinga, J. J. (1975). Biofeedback for chemical problem patients: A developmental process. Journal of Biofeedback, 2, 17-27.

Graap, K., \& Freides, D. (1998). Regarding the database for the Peniston alpha-theta EEG biofeedback protocol. Applied Psychophysiology and Biofeedback, 23, 265-272.

Graap, K., Ready, D. J., Freides, D., Daniels, B., \& Baltzell, D. (1997). EEG biofeedback treatment for Vietnam veterans suffering from posttraumatic stress disorder. Journal of Neurotherapy, 2, 65-66.

Grabowski, J., Shearer, J., Merrill, J., \& Negus, S. S. (2004). Agonistlike, replacement pharmacotherapy for stimulant abuse and dependence. Addictive Behaviors, 29, 1439-1464.

Grant, B. F., Stinson, F., Dawson, D. A., Chou, S. P., Ruan, W. J., \& Pickering, R. P. (2004). Co-occurrence of 12-month alcohol and drug use disorders and personality disorders in the United States. Archives of General Psychiatry, 61, 361-368.

Green, E. E., Green, A. M., \& Walters, E. D. (1974). Alpha-theta biofeedback training. Journal of Biofeedback, 2, 7-13.

Gritz, E. R., Shiffman, S. M., Jarvik, M. E., Haber, J., Dymond, A. M., Coger, R., Charuvastra, V., \& Schlesinger, J. (1975). Physiological and psychological effects of methadone in man. Archives of General Psychiatry, 2(2), 237-242.

Hada, M., Porjesz, B., Begleiter, H., \& Polich, J. (2000). Auditory P3a assessment of male alcoholics. Biological Psychiatry, 48, 276286.

Hammond, D. C. (2006). What is neurofeedback? Journal of Neurotherapy, 10(4), 25-36.

Herning, R. I., Glover, B. J., \& Guo, X. (1994a) Effects of cocaine on P3B in cocaine abusers. Neuropsychobiology, 30, 132-142.

Herning, R. I., Glover, B. J., Koeppl, B., Phillips, R. L., \& London, E. D. (1994b). Cocaine induced increases in EEG alpha and beta activity: Evidence for reduced cortical processing. Neuropsychopharmacology, 11, 1-9.

Herning, R. I., Jones, R. T., Hooker, W. D., Mendelson. J., \& Blackwell, L. (1985). Cocaine increases EEG beta: A replication and extension of Hans Berger's historic experiments. Electroencephalography and Clinical Neurophysiology, 60(6), 470477.

Hill, H. E. (1962). The social deviant and initial addiction to narcotics and alcohol. Quarterly Journal of Studies on Alcohol, 23, 562582.

Hockman, C. H., Perrin, R. G., \& Kalant, H. (1971). Electroencephalographic and behavioral alterations produced by delta-9tetrahydrocannabinol. Science, 172, 968-970.

Horner, B. R., \& Scheibe, K. E. (1997). Prevalence and implications of attention-deficit hyperactivity disorder among adolescents in treatment for substance abuse. Journal of the American Academy of Child and Adolescent Psychiatry, 36(1), 30-36.

Huang-Storms, L., Bodenhamer-Davis, E., Davis, R., \& Dunn, J. (2006). QEEG-guided neurofeedback for children with histories of abuse and neglect: Neurodevelopmental rationale and pilot study. Journal of Neurotherapy, 10(4), 3-16.

Hughes, J. R., \& John, E. R. (1999). Conventional and quantitative electroencephalography in psychiatry. Journal of Neuropsychiatry and Clinical Neuroscience, 11, 190-208.

Jacobsen, L. K., Southwick, S., \& Kosten, T. R. (2001). Substance use disorders in patients with posttraumatic stress disorder. American Journal of Psychiatry, 158, 1184-1190.

Jeffcoat, A. R., Perez-Reyes, M., Hill, J. M., Sadler, B. M., \& Cook, C. E. (1989). Cocaine disposition in humans after intravenous injection, nasal insufflation (snorting), or smoking. Drug Metabolism Disposition, 17, 153-159.
Jones, F. W., \& Holmes, D. S. (1976). Alcoholism, alpha production, and biofeedback. Journal of Consulting and Clinical Psychology, 44(2), 224-228.

Iwanami, A., Kuroki, N., Iritani, S., Isono, H., Okajima, Y., \& Kamijima, K. (1998). P3a of event-related potential in chronic methamphetamine dependence. Journal of Nervous and Mental Diseases, 186, 746-751.

Iwanami, A., Suga, I., Kaneko, T., Sugiyama, A., \& Nakatani, Y. (1994). P300 component of event-related potentials in methamphetamine psychosis and schizophrenia. Progress in Neuropsychopharmacology and Biological Psychiatry, 18, 465-475.

Kalechstein, A. D., Newton, T. F., \& Green, M. F. (2003). Methamphetamine dependence is associated with neurocognitive impairment in the initial phases of abstinence. Journal of Neuropsychiatry \& Clinical Neurosciences, 15, 215-220.

Kalechstein, A. D., Newton, T. F., Longshore, D., Anglin, M. D., van Gorp, W. G., \& Gawin, F. H. (2000). Psychiatric comorbidity of methamphetamine dependence in a forensic sample. Journal of Neuropsychiatry \& Clinical Neurosciences, 12, 480-484.

Kaiser, D. A. (2006). What is quantitative EEG? Journal of Neurotherapy, 10(4), 37-52.

Kaiser, D. A., \& Othmer, S. (2000). Effect of Neurofeedback on variables of attention in a large multi-center trial. Journal of Neurotherapy, 4(1), 5-15.

Kaplan, R. F., Glueck, B. C., Hesselbrock, M. N., \& Reed, H. B. (1985). Power and coherence analysis of the EEG in hospitalized alcoholics and nonalcoholic controls. Journal of Studies on Alcohol, 46, 122-127.

Kaplan, R., Hesselbrock, V., O’Connor, S., \& Depalma, N. (1988). Behavioral and EEG Responses to alcohol in nonalcoholic men with a family history of alcoholism. Progress in NeuroPharmacology \& Biological Psychiatry, 12, 873-885.

Katayama, J., \& Polich, J. (1996). P300 from one-, two-, and threestimulus auditory paradigms. International Journal of Psychophysiology, 23, 33-40.

Katayama, J., \& Polich, J. (1998). Stimulus context determines P3a and P3b. Psychophysiology, 35, 23-33.

Kelly, M. J. (1997). Native Americans, neurofeedback, and substance abuse theory: Three year outcome of alpha/theta neurofeedback training in the treatment of problem drinking among Dine' (Navajo) People. Journal of Neurotherapy, 2(3), 24-60.

Khantzian, E. J. (1985). The self-medication hypothesis of addictive disorders: Focus on heroin and cocaine dependence. American Journal of Psychiatry, 142, 1259-1264.

Khantzian, E. J. (1997). The self-medication hypothesis of substance use disorders: A reconsiderations and recent applications. Harvard Review Psychiatry, 4, 231-244.

Knight, R. T. (1984). Decreased response to novel stimuli after prefrontal lesions in man. Electroencephalography \& Clinical Neurophysiology, 59, 9-20.

Koob, G. F., \& Le Moal, M. (2001). Drug addiction, dysregulation of reward, and allostasis. Neuropsychopharmacology, 24, 97129.

Kouri, E. M., Lukas, S. E., \& Mendelson, J. H. (1996). P300 assessment of opiate and cocaine abusers: Effects of detoxification and bupernorphine treatment. Biological Psychiatry, 40, 617-628.

Lamontagne, Y., Beausejour, R., Annable, L., \& Tetreault, L. (1977). Alpha and EMG feedback training in the prevention of drug abuse. A controlled study. Canadian Psychiatry Association Journal, 22(6), 301-310.

Landen, M. G., Castle, S., Nolte, K. B., Gonzales, M., Escobedo, L., Chatterjee, B., Johnson, K., \& Sewell, C. M. (2003). Methodological issues in the surveillance of poisoning, illicit drug 
overdose, and heroin overdose deaths in New Mexico. American Journal of Epidemiology, 157, 273-278.

LaVaque, T. J., Hammond, D. C., Trudeau, D., Monastra, V. J., Perry, J., \& Lehrer, P. (2002). Template fordeveloping guidelines for the evaluation of the clinical efficacy of psychophysiological interventions. Applied Psychophysiology and Biofeedback, 27(4), 273-281.

Levin, F. R., \& Kleber, H. D. (1995). Attention-deficit hyperactivity disorder and substance abuse: Relationships and implications for treatment. Harvard Review of Psychiatry, 2(5), 246-58.

Lowe, F. (1999). How essential is the EEG component of the Peniston and Kulkosky protocol? Applied Psychophysiology and Biofeedback, 24(2), 117-118.

Lukas, S. E. (1991). Topographic mapping during cocaine-induced intoxication and self-administration. In G. Racagni, N. Brunello, \& T. Fukuda (Eds.), Biological psychiatry (Vol. 2, pp. 25-29). New York: Elsevier Science Publishers.

Lukas, S. E. (1993). Advanced electrophysiological imaging techniques for studying drug effects. In E. D. London (Ed.), Imaging Drug action in the brain (Chapter 15, pp. 389-404). Boca Raton, FL: CRC Press.

Lukas, S. E., Mendelson, J. H., Amass, L., \& Benedikt, R. (1989). Behavioral and EEG studies of acute cocaine administration: comparisons with morphine, amphetamine, pentobarbital, nicotine, ethanol and marijuana. NIDA Research Monographs, 95, $146-151$.

Lukas, S. E., Mendelson, J. H., \& Benedikt, R. (1995). Electroencephalographic correlates of marihuana-induced euphoria. Drug and Alcohol Dependence, 37, 131-140.

Maldonado, R. (1997). Participation of noradrenergic pathways in the expression of opiate withdrawal: Biochemical and pharmacological evidence. Neuroscience Biobehavoral Reviews, 21(1), 91-104.

Manuzza, S., Klein, R. G., Konig, P. H., \& Giampino, P. L. (1989). Hyperactive boys almost grown up, IV. criminality and its relationship to psychiatric status. Archives of General Psychiatry, 46, 1073-1079.

Manuzza, S., Klein, R. G., Bessler, A., Malloy, P., \& LaPadula, M. (1998). Adult psychiatric status of hyperactive boys grown up. American Journal of Psychiatry, 155(4), 493-498.

Mark, T. L., Woody, G. E., Juday, T., \& Kleber, H. D. (2001). The economic costs of heroin addiction in the United States. Drug \& Alcohol Dependence, 61, 195-206.

Meyerstein, A. N. (1964). Drug addiction: A review. The Journal of School Health, 34, 77-87.

Michael, A., Mirza, K. A., Mukundan, C., \& Channabasavanna, S. M. (1993). Interhemispheric electroencephalographic coherence as a biological marker in alcoholism. Acta Psychiatrica Scandinavica, 87, 213-217.

Miller, W., \& Rollnick, S. (2002). Motivational interviewing. NY: Guilford.

Monastra, V. J., Lynn, S., Linden, M., Lubar, J. F., Gruzelier, J., \& LaVaque, T. J. (2005). Electroencephalographic biofeedback in the treatment of attention-deficit/hyperactivity disorder. Applied Psychophysiology and Biofeedback, 30, 95-114.

Moore, J. P., \& Trudeau, D. L. (1998). Alpha theta brainwave biofeedback is not specific to the production of theta/alpha crossover and visualizations. Journal of Neurotherapy, 3(1), 63.

Myers, R., \& Privette, T. (1989). A Neuroanatomical substrate for alcohol drinking: Identification of Tetrahydropapeveroline (THP)-reactive sites in the rat brain. Brain Research Bulletin, 22, 899-911.

Näätänen, R. (1990). The role of attention in auditory information processing as revealed by event-related potentials and other brain measures of cognitive functioning. Behavioral \& Brain Sciences, 13, 201-287.
Newton, T. F., Cook, I. A., Kalechstein, A. D., Duran, S., Monroy, F., Ling, W., \& Leuchter, A. F. (2003). Quantitative EEG abnormalities in recently abstinent methamphetamine-dependent individuals. Clinical Neurophysiology, 114, 410-415.

Newton, T. F., Kalechstein, A. D., Hardy, D. J., Cook, I. A., Nestor, L., Ling, W., \& Leuchter, A. F. (2004). Association between quantitative EEG and neurocognition in methamphetaminedependent volunteers. Clinical Neurophysiology, 115, 194-198.

Niedermeyer, E. (1999). Sleep and the EEG. In E. Niedermeyer \& F. Lopes Da Silva (Eds.), Electroencephalography: Basic principles, clinical applications, and related fields (4th ed., pp. 174189). Baltimore: Williams and Wilkins.

Niedermeyer, E., \& Lopes da Silva, F. (1982). Electroencephalography. Basic principles, clinical applications and related fields. Baltimore: Urban and Schwarzenberg.

Noldy, N. E., \& Carlen, P. L. (1997). Event-related potential changes in cocaine withdrawal: Evidence for long-term cognitive effects. Neuropsychobiology, 36, 53-56.

Noldy, N. E., Santos, C. V., Politzer, N., Blair, R. D., \& Carlen, P. L. (1994). Quantitative EEG changes in cocaine withdrawal: Evidence for long-term CNS effects. Neuropsychobiology, 30, 189-196.

Nowlis, D. P., \& Kamiya, J. (1970). The control of electroencephalograhic alpha rhythms through auditory feedback and the associated mental activity. Psychophysiology, 6, 476-484.

O’Brien, C. P., Charney, D. S., Lewis, L., Cornish, J. W., Post, R., et al. (2004). Priority actions to improve the care of persons with co-occurring substance abuse and other mental disorders: A call to action. Biological Psychiatry, 56, 703-713.

O'Connor, S., Bauer, L. O., Tasman, A., \& Hesselbrock, V. M. (1994). Reduced P3 amplitudes of ERPs are associated with both a family history of alcoholism and antisocial personality disorder. Progress in Neuropsychopharmacology and Biological Psychiatry, 18, 1307-1321.

O’Leary, D. S., Block, R. I., Koeppel, J. A., Flaum, M., Schultz, S., Andreasen, N. C., Ponto, L., Watkins, G. L., Hurtig, R. R., \& Hichwa, R. D. (2002). Effects of smoking marijuana on brain perfusion and cognition. Neuropsychopharmacology, 26, 802-816.

Olivennes, A., Charles-Nicolas, A., \& Olievenstein, C. I. (1983). Altérations de l'électroencéphalogramme de veille dans la grande héroïnomanie. Annals Medical Psychologie (Paris), 141, 458-469.

Papageorgiou, C., Liappas, I., Asvestas, P., Vasios, C., Matsopoulos, G. K., Nikolaou, C., Nikita, K. S., Usunoglu, N., \& Rabavilas, A. (2001). Abnormal P600 in heroin addicts with prolonged abstinence elicited during a working memory test. NeuroReport, 12, 1773-1778.

Papageorgiou, C., Liappas, I., Ventouras, E. M., Nikolaou, C., Kitsonas, E. N., Uzunoglu, N., \& Rabavilas, A. D. (2004). Longterm abstinence syndrome in heroin addicts: Indices of P300 alterations associated with a short memory task. Progress in Neuropsychopharmacology and Biological Psychiatry, 28, 1109-1115.

Passini, F. T., Watson, C. G., Dehnel, L., Herder, J., \& Watkins, B. (1977). Alpha wave biofeedback training therapy in alcoholics. Journal of Clinical Psychology, 33(1), 292-299.

Patrick, G., Straumanis, J. J., Struve, F. A., Fitz-Gerald, M. J., \& Manno, J. E. (1997). Early and middle latency evoked potentials in medically and psychiatrically normal daily marihuana users: A paucity of significant findings. Clinical Electroencephalography, 28(1), 26-31.

Patrick, G., Straumanis, J. J., Struve, F., Nixon, F., Fitz-Gerald, M. J., Manno, J. E., \& Soucair, M. (1995). Auditory and visual P300 event related potentials are not altered in medically and psychiatrically normal chronic marihuana users. Life Sciences, $56,2135-2140$. 
Peniston, E. G. (1998). Comments by Peniston. Applied Psychophysiology and Biofeedback, 23, 273-275.

Peniston, E. G., \& Kulkosky, P. J. (1989). Alpha-theta brainwave training and beta endorphin levels in alcoholics. Alcoholism Clinical and Experimental Research, 13, 271-279.

Peniston, E. G., \& Kulkosky, P. J. (1990). Alcoholic personality and alpha-theta brainwave training. Medical Psychotherapy, 2, 3755.

Peniston, E. G., \& Kulkosky, P. G. (1991). Alpha-theta brain wave neurofeedback for Vietnam veterans with combat related post traumatic stress disorder. Medical Psychotherapy, 4, 1-14.

Peniston E. G., Marriman, D. A., Deming, W. A., \& Kulkosky, P. G. (1993). EEG alpha-theta brain wave synchronization in Vietnam theater veterans with combat related post traumatic stress disorder and alcohol abuse. Medical Advances in Medical Psychotherapy, 6, 37-50.

Polich, J. (1990). P300, probability, and interstimulus interval. Psychophysiology, 27, 396-403.

Polich, J., Pollock, V. E., \& Bloom, F. E. (1994). Meta-analysis of P300 from males at risk for alcoholism. Psychological Bulletin, 115(1), 55-73.

Polich, J., \& Herbst, K. L. (2000). P300 as a clinical assay: Rationale, evaluation and findings. International Journal of Psychophysiology, 38(1), 3-19.

Pollock, V. E., Schneider, L. S., Zemansky, M. F., Gleason, R. P., \& Pawluczyk, S. (1992). Topographic quantitative EEG amplitude in recovered alcoholics. Psychiatry Research, 45, 25-32.

Polunina, A. G., \& Davydov, D. M. (2004). EEG spectral power and mean frequencies in early heroin abstinence. Progress in Neuropsychopharmacology and Biological Psychiatry, 28(1), 73-82.

Porjesz, B., \& Begleiter, H., (1998). Genetic basis of event-related potentials and their relationship to alcoholism and alcohol use. Journal of Clinical Neurophysiology, 15, 44-57.

Porjesz, B., Almasy, L., Edenberg, H. J., Wang, K., Chorlian, D. B., Foroud, T., Goate, A., Rice, J. P., O'Connor, S. J., Rohrbaugh, J., Kuperman, S., Bauer, L. O., Crowe, R. R., Schuckit, M. A., Hesselbrock, V., Conneally, P. M., Tischfield, J. A., Li, T. K., Reich, T., \& Begleiter, H. (2002). Linkage disequilibrium between the beta frequency of the human EEG and a GABAA receptor gene locus. Proceedings National Academy of Sciences of the USA, 99(6), 3729-3733.

Porjesz, B., Rangaswamy, M., Kamarajan, C., Jones, K. A., Padmanabhapillai, A., \& Begleiter, H. (2005). The utility of neurophysiological markers in the study of alcoholism. Clinical Neurophysiology, 116, 993-1018.

Prichep, L. S., Alper, K. A., Kowalik, S. C., \& Rosenthal, M. (1996). Neurometric QEEG studies of crack cocaine dependence and treatment outcome. Journal of Addictive Disorders, 15(4), 3953.

Prichep, L. S., Alper, K. R., Kowalik, S. C., Vaysblat, L. S., Merkin, H. A., et al. (1999). Prediction of treatment outcome in cocaine dependent males using quantitative EEG. Drug and Alcohol Dependence, 54, 35-43.

Prichep, L. S., Alper, K. A., Sverdlov, L., Kowalik, S. C., John, E. R., Merkin, H., Tom, M. L., \& Rosenthal, M. (2002). Outcome related electrophysiological subtypes of cocaine dependence. Clinical Electroencephalography, 33(1), 8-20.

Pritchard, W. (1981). Psychophysiology of P300. Psychological Bulletin, 89, 506-540.

Pritchard, W. S. (1986). Cognitive event-related potential correlates of schizophrenia. Psychological Bulletin, 100, 43-66.

Pritchard, W. S., Sokhadze, E. M., \& Houlihan, M. (2004). Effects of nicotine and smoking on even-related potentials: A review. Nicotine \& Tobacco Research, 6(4), 961-984.
Rangaswamy, M., Porjesz, B., Chorlian, D., Wang, K., Jones, K., Bauer, L. O., Rohrbaugh, J., O'Connor, S. J., Kuperman, S., Reich, T., \& Begleiter, H. (2002). Beta power in the EEG of alcoholics. Biological Psychiatry, 52, 831-842.

Rangaswamy, M., Porjesz, B., Chorlian, D., Wang, K., Jones, K., Kuperman, S., Rohrbaugh, J., O'Connor, S. J., Bauer, L. O., Reich, T., \& Begleiter, H. (2004). Resting EEG in offspring of male alcoholics: Beta frequencies. International Journal of Psychophysiology, 51, 239-251.

Regier, D. A., Farmer, M. E., Rae, D. S., Locke, B. Z., Keith, S. J., Judd, L. L., \& Goodwin, F. K. (1990). Comorbidity of mental disorders with alcohol and other drug abuse: Results from the Epidemiologic Catchment Area (ECA) Study. Journal of the American Medical Association, 264, 2511-2518.

Reid, M., Flammino, F., Howard, B., Nilsen, D., \& Prichep, L. S. (2006). Topographic imaging of quantitative EEG in response to smoked cocaine self-administration in humans. Neuropsychopharmacology, 31, 872-884.

Rodin, E. A., Domino, E. F., \& Porzak, J. P. (1970). The marihuanainduced 'social high'. Neurological and electroencephalographic concomitants. Journal of American Medical Association, 213(8), $1300-1302$.

Roemer, R. A., Cornwell, A., Dewart, D., Jackson, P., \& Ercegovac, D. V. (1995). Quantitative electroencephalographic analysis in cocaine-preferring polysubstance abusers during abstinence. Psychiatry Research, 58, 247-257.

Romano-Micha, J. (2003). Databases or specific training protocols for neurotherapy? A proposal for a clinical approach to neurotherapy. Journal of Neurotherapy, 7(3,4), 69-85.

Roth, W. T., Galanter, M., Weingartner, H., Vaughan, T. B., \&. Wyatt, R. J. (1973). Marijuana and synthetic 9-trans-tetrahydrocannabinol: Some effects on the auditory evoked response and background EEG in humans. Biological Psychiatry, 6(3), 221-233.

Ryabinin, A., \& Weitemier, A. (2006). The urocortin 1 neurocircuit: Ethanol-sensitivity and potential involvement in alcohol consumption. Brain Research Reviews, 52, 368-380.

Saletu, B., Anderer, P., Saletu-Zyhlarz, G. M., Arnold, O., \& PascualMarqui, R. D. (2002). Classification and evaluation of the pharmacodynamics of psychotropic drugs by single-lead pharmaco-EEG, EEG mapping and tomography (LORETA). Methods and Findings in Experimental and Clinical Pharmacology, 24(Suppl. C), 97-120.

Saletu-Zyhlarz, G. M., Arnold, O., Anderer, P., Oberndorfer, S., Walter, H., Lesch, O. M., Boning, J., \& Saletu, B. (2004). Differences in brain function between relapsing and abstaining alcohol-dependent patients, evaluated by EEG mapping. Alcohol Alcoholism, 39, 233-240.

Samochowiec, J., Mazur-Kucharska, J., Grywacz, A., Jablonski, M., Rommelspacher, H., et al. (2006). Family-based and casecontrol study of DRD2, DAT, 5HTT, COMT genes polymorphisms in alcohol dependence. Neuroscience Letters, 410, 1-5.

Saxby, E., \& Peniston, E. G. (1995). Alpha-theta brainwave neurofeedback training: An effective treatment for male and female alcoholics with depressive symptoms. Journal of Clinical Psychology, 51(5), 685-693.

Scott, W. C., Brod, T. M., Sideroff, S., Kaiser, D., \& Sagan, M. (2002). Type-specific EEG biofeedback improves residential substance abuse treatment. Paper presented at American Psychiatric Association Annual Meeting 2002.

Scott, W., \& Kaiser, D. (1998). Augmenting chemical dependency treatment with neurofeedback training. Journal of Neurotherapy, $3(1), 66$.

Scott, W. C., Kaiser, D., Othmer, S., \& Sideroff, S. I. (2005). Effects of an EEG biofeedback protocol on a mixed substance abusing 
population. American Journal of Drug Alcohol Abuse, 31(3), 455-469.

Schneider, F., Elbert, T., Heimann, H., Welker, A., Stetter, F., Mattes, R., Birbaumer, N., \& Mann, K. (1993). Self-regulation of slow cortical potentials in psychiatric patients: Alcohol dependency. Biofeedback \& Self-Regulation, 18(1), 23-32.

Schubiner, H., Tzelepis, A., Milberger, S., Lockhart, N., Kruger, M., Kelley, B. J., \& Schoener, E. P. (2000). Prevalence of attentiondeficit/hyperactivity disorder and conduct disorder among substance abusers. Journal Clinical Psychiatry, 61, 244-251.

Schuckit, M. A., \& Smith, T. L. (1996). An 8-year follow-up of 450 sons of alcoholic and control subjects. Archives of General Psychiatry, 53, 202-210.

Sheehan, M. F. (1993). Dual diagnosis. Psychiatry Quarterly, 64, 107-134.

Shufman, E., Perl, E., Cohen, M., Dickman, M., Gandaku, D., Adler, D., Veler, A., Bar-Hamburger, R., \& Ginath, Y. (1996). Electroencephalography spectral analysis of heroin addicts compared with abstainers and normal controls. Israel Journal of Psychiatry Related Science, 33, 196-206.

Sim, M. K. (1976). Treatment of disease without the use of drugs I. Research on biofeedback training. Singapore Medical Journal, 17(3), 167-173.

Simon, S. L., Domier, C., Sim, T., Richardson, K., Rawson, R., \& Ling, W. (2002). Cognitive performance of current methamphetamine and cocaine abusers. Journal of Addictive Diseases, $21,61-74$.

Simon, S. L., Dacey, J., Glynn, S., Rawson, R., \& Ling, W. (2004). The effect of relapse on cognition in abstinent methamphetamine abusers. Journal of Substance Abuse Treatment, 27, 59-66.

Skok, A., Shubina, O., Finkelberg, A., Shtark, M., \& Jafarova, O. (1997). EEG training in the treatment of addictive disorders. Applied Psychophysiology \& Biofeedback, 22, 130.

Sokhadze, T (2005). Neurofeedback and cognitive behavioral therapy based intervention in dual diagnosis: A neurobiological model. Journal of Neurotherapy, 9(4), 123-124.

Sokhadze, E. M., Martin, L. E., Stotts, A. L., \& Potts, G. F. (2004). Motivational Interviewing enhances cognitive ERPs in a selective attention task in cocaine addicts. Society for Neurosciences 34th Annual Meeting, San Diego, CA.

Sokhadze, E. M., Potts, G. F., Martin, L. E., \& Stotts, A. L. (2005). Motivational Interviewing enhances performance and ERPs in a selective attention task in cocaine addicts. Applied Psychophysiology \& Biofeedback, 30(4), 406-407.

Sokhadze, T., Stewart, C. M., \& Hollifield, M. (2007). Integrating cognitive neuroscience research and cognitive behavioral treatment with neurofeedback therapy in drug addiction comorbid with Posttraumatic Stress Disorder: A conceptual review. Journal of Neurotherapy, 11(2), 13-44.

Sokhadze, T., Tasman, A., Stewart, C. M., Singh, S., \& Hollifield, M. (2007). Dense-array QEEG/ERP study of frontal deficits in patients with substance use disorder and PTSD. Presented at the 15th Annual Conference of ISNR, San Diego, CA, September $6-9$.

Stormark, K. M., Laberg, J. C., Nordby, H., \& Hugdahl, K. (2000). Alcoholics' selective attention to alcohol stimuli: Automated processing? Journal of Studies on Alcohol, 61, 18-23.

Stotts, A. L., Potts, G. F., Ingersoll, G., George, M. R., \& Martin, L. E. (2006). Preliminary feasibility and efficacy of a brief motivational intervention with psychophysiological feedback for cocaine abuse. Substance Abuse, 27(4), 9-20.

Struve, F. A., Patrick, G., Straumanis, J. J., Fitz-Gerald, M. J., \& Manno, J. (1998). Possible EEG sequelae of very long duration marihuana use: Pilot findings from topographic quantitative EEG analyses of subjects with 15 to 24 years of cumulative daily exposure to THC. Clinical Electroencephalography, 29(1), 31-36.
Struve, F. A., Straumanis, J. J., \& Patrick, G. (1994). Persistent topographic quantitative EEG sequelae of chronic marihuana use: A replication study and initial discriminant function analysis. Clinical Electroencephalography, 25, 63-75.

Struve, F. A., Straumanis, J. J., Patrick, G., Leavitt, J., Manno, J., \& Manno, B. R. (1999). Topographic quantitative EEG sequelae of chronic marihuana use: A replication using medically and psychiatrically screened normal subjects. Drug and Alcohol Dependence, 56, 167-179.

Struve, F. A., Straumanis, J. J., Patrick, G., \& Price, L. (1989). Topographic mapping of quantitative EEG variables in chronic heavy marihuana users: Empirical findings with psychiatric patients. Clinical Electroencephalography, 20(1), 6-23.

Struve, F. A., Manno, B. R., Kemp, P., Patrick, G., \& Manno, J. (2003). Acute marihuana (THC) exposure produces a "transient" topographic quantitative EEG profile identical to the "persistent" profile seen in chronic heavy users. Clinical Electroencephalography, 34, 75-83.

Substance Abuse and Mental Health Services Administration (2004). Results from the 2003 National Survey on drug use and health (NSDUH Series H-25), Office of Applied Studies, Substance Abuse and Mental Health Services Administration, Rockville, MD.

Substance Abuse and Mental Health Services Administration (2006). Results from the 2005 National Survey on Drug Use and health: National Findings (Office of Applied Studies, NSDUH Series H30, DHHS Publication No. SMA 06-4194). Rockville, MD.

Swartz, J. A., \& Lurigio, A. J. (1999). Psychiatric illness and comorbidity among adult male jail detainees in drug treatment. Psychiatry Service, 50, 1628-1630.

Tapert, S. F., Ozyurt, S. S., Myers, M. G., \& Brown, S. A. (2004). Neurocognitive ability in adults coping with alcohol and drug relapse temptations. American Journal Drug Alcohol Abuse, 30(2), 445-460.

Tarbox, A. R. (1983). Alcoholism, biofeedback and internal scanning. Journal Study of Alcohol, 44(2), 246-261.

Tarter, R. E., Kirisci, L., Mezzich, A., Cornelius, J., Pajer, K., Vanyukov, M. M., Gardner, T., Blackson, D., \& Clark, D. (2003). Neurobehavior disinhibition in childhood predicts early age at onset of substance disorder. American Journal of Psychiatry, 160, 1078-1085.

Tassinari, C. A., Ambrosetto, H. G., Peraita-Adrados, M. P., \& Gastaut, H. (1976). The neuropsychiatric syndrome of delta-9tetrahydrocannabinol and cannabis intoxication in naïve subjects: A clinical and polygraphic study during wakefulness and sleep. In M. C. Braude \& S. Szara (Eds.), The Pharmacology of marihuana (pp. 357-382). New York: Raven Press.

Taub, E., \& Rosenfeld, J. P. (1994). Is alpha/theta training the effective component of the alpha/theta therapy package for the treatment of alcoholism? Biofeedback, 22(3), 12-14.

Thatcher, R. W., Walker, R. A., Gerson, I., \& Geisler, F. (1989). EEG discriminant analysis of mild head trauma. EEG and Clinical Neurophysiology, 73, 93-10.

Triffleman, E., Carroll, K., \& Kellogg, S. (1999). Substance dependence posttraumatic stress therapy: An integrated cognitive-behavioral approach. Journal of Substance Abuse Treatment, 17, 3-14.

Trudeau, D. L., Thuras, P., \& Stockley, H. (1999). Quantitative EEG findings associated with chronic stimulant and cannabis abuse and ADHD in an adult male substance use disorder population. Clinical Electroencephalography, 30, 165-174.

Trudeau, D. L. (2000). A review of the treatment of Addictive Disorders by EEG biofeedback. Clinical Electroencephalography, 31, 13-26.

Trudeau, D. L. (2005a). EEG biofeedback for addictive disordersThe state of the art in 2004. Journal of Adult Development, 12, 139-146. 
Trudeau, D. L. (2005b). Applicability of brain wave biofeedback to substance use disorder in adolescents. Child and Adolescent Clinics of North America, 14, 125-136.

Twemlow, S. W., \& Bowen, W. T. (1976). EEG Biofeedback induced self actualization in alcoholics. Journal of Biofeedback, $3,20-25$.

Twemlow, S. W., \& Bowen, W. T. (1977). Sociocultural predictors of self actualization in EEG biofeedback treated alcoholics. Psychological Reports, 40, 591-598.

Twemlow, S. W., Sizemore, D. G., \& Bowen, W. T. (1977). Biofeedback induced energy redistribution in the alcoholic EEG. Journal of Biofeedback, 3, 14-19.

Uhl, G., Blum, K., Noble, E., \& Smith, S. (1993). Substance abuse vulnerability and D2 receptor genes. Trends Neuroscience, 16, 83-88.

Van Beijsterveldt, C. E. M., \& Van Baal, G. C. M. (2002). Twin and family studies of the human electroencephalogram: A review and a meta-analysis. Biological Psychology, 61, 111-138.

Van den Brink, W., \& van Ree, J. M. (2003). Pharmacological treatments for heroin and cocaine addictions. European Neuropsychopharmacology, 13, 476-487.

Vanathy, S., Sharma, P. S. V. N., \& Kumar, K. B. (1998). The efficacy of alpha and theta neurofeedback training in treatment of generalized anxiety disorder. Indian Journal of Clinical Psychology, 25, 136-143.

Vernon, D., Egner, T., Cooper, N., Compton, T., Neilands, C., Sheri, A., \& Gruzelier, J. (2003). The effect of training distinct neurofeedback protocols on aspects of cognitive performance. International Journal of Psychophysiology, 47, 75-85.

Vernon, D. J. (2005). Can neurofeedback training enhance performance? An evaluation of the evidence with implication for future research. Applied Psychophysiology \& Biofeedback, 30, 347-364.

Vanderschuren, L. J., \& Everitt, B. J. (2004). Drug seeking becomes compulsive after prolonged cocaine self-administration. Science, $305,1017-1019$.

Venneman, S., Leuchter, A., Bartzokis, G., Beckson, M., Simon, S. L., Schaefer, M., Rawson, R., Newton, T., Cook, I. A., Uijtdehaage, S., \& Ling, W. (2006). Variation in neurophysiological function and evidence of quantitative electroencephalogram discordance: Predicting cocaine-dependent treatment attrition. Journal of Neuropsychiatry Clinical Neurosciences, 18(2), 208-216.

Vocci, F., \& Ling, W. (2005). Medications development: Successes and challenges. Pharmacology \& Therapeutics, 108, 94-108.

Volavka, J., Crown, P., Dornbush, R., Feldstein, S., \& Fink, M. (1973). EEG, heart rate and mood change ('high') after cannabis. Psychopharmacologia, 32, 11-25.
Volavka, J., Dornbush, R., Feldstein, S., Clare, G., Zaks, A., Fink, M., \& Freedman, A. M. (1971). Marihuana, EEG and behavior. Annals of New York Academy of Sciences, 191, 206-215.

Volkow, N. D., Fowler, J. S., \& Wang, G. J. (2003). The addicted human brain: Insights from imaging studies. Journal of Clinical Investigations, 111, 1444-1451.

Volkow, N. D., Fowler, J. S., \& Wang, G. J. (2004). The addicted human brain viewed in the light of imaging studies: Brain circuits and treatment strategies. Neuropharmacology, 47, 3-13.

Volkow, N. D., \& Li, T. K. (2005). Drugs and alcohol: Treating and preventing abuse, addiction and their medical consequences. Pharmacology \& Therapeutics, 108, 3-17.

Vos, H. (1989). Denial of the inner reality: Observations on drug abuse and addiction based on psychotherapies after treatment in a therapeutic community in the Netherlands. Journal of Substance Abuse Treatment, 6, 193-199.

Walters, D. (1998). EEG neurofeedback treatment for alcoholism. Biofeedback, 26, 18-21.

Watson, C.G, Herder, J., \& Passini, F. T. (1978). Alpha biofeedback therapy in alcoholics: An 18-month follow-up. Journal of Clinical Psychology, 34(3), 765-769.

Wender, P. H. (1995). Attention-deficit hyperactivity disorder in adults. New York: Oxford University Press.

Wert, R. C., \& Raulin, M. L. (1986). The chronic cerebral effects of cannabis use. I. Methodological issues and neurological findings. International Journal of Addiction, 21, 605-628.

Whitlow, C. T., Freedland, C. S., \& Porrino, J. L. (2002). Metabolic mapping of the time-dependent effects of delta 9-tetrahydrocannabinol administration in the rat. Psychopharmacology, 161, 129-136.

Wilens, T. E., Biederman, J., \& Mick, E. (1998). Does ADHD affect the course of substance abuse? Findings from a sample of adults with and without ADHD. American Journal of Addictions, 7(2), 156-163.

Winterer, G., Enoch, M. A., White, K., Saylan, M., Coppola, R., \& Goldman, D. (2003a). EEG phenotype in alcoholism: Increased coherence in the depressive subtype. Acta Psychiatrica Scandinavica, 108, 51-60.

Winterer, G., Kloeppel, B., Heinz, A., Ziller, M., Dufeu, P., Schmidt, L. G., \& Herrmann, W. M. (1998). Quantitative EEG (QEEG) predicts relapse in patients with chronic alcoholism and points to a frontally pronounced cerebral disturbance. Psychiatry Research, 78, 101-113.

Winterer, G., Smolka, M., Samochowiec, J., Ziller, M., Mahlberg, R., Gallinat, J., Rommelspacher, H., Herrmann, W., \& Sander, T. (2003b). Association of EEG coherence and an exonic $\mathrm{GABA}_{\mathrm{B}} \mathrm{R} 1$ gene polymorphism. American Journal of Medical Genetics, 117B, 51-56. 